\title{
CO 1st overtone spectra of cool evolved stars: Diagnostics for hydrodynamic atmosphere models ${ }^{\star}$
}

\author{
J. H. Bieging, M. J. Rieke, and G. H. Rieke
}

\author{
Steward Observatory, The University of Arizona, Tucson AZ 85721, USA
}

Received 4 October 2001 / Accepted 11 January 2002

\begin{abstract}
We present spectra covering the wavelength range 2.28 to $2.36 \mu \mathrm{m}$ at a resolution of $\Delta \lambda=0.0007 \mu \mathrm{m}$ (or $R=3500$ ) for a sample of 24 cool evolved stars. The sample comprises $8 \mathrm{M}$ supergiants, $5 \mathrm{M}$ giants, $3 \mathrm{~S}$ stars, 6 carbon stars, and 2 RV Tauri variables. The wavelengths covered include the main parts of the ${ }^{12} \mathrm{C}^{16} \mathrm{O} v=$ $2-0$ and $3-1$ overtone bands, as well as the $v=4-2$ and ${ }^{13} \mathrm{CO} v=2-0$ bandhead regions. CO lines dominate the spectrum for all the stars observed, and at this resolution most of the observed features can be identified with individual CO $R$ - or $P$-branch lines or blends. The observed transitions arise from a wide range of energy levels extending from the ground state to $E / k>20000 \mathrm{~K}$. We looked for correlations between the intensities of various $\mathrm{CO}$ absorption line features and other stellar properties, including IR colors and mass loss rates. Two useful CO line features are the $v=2-0$ R14 line, and the CO $v=2-0$ bandhead. The intensity of the 2-0 bandhead shows a trend with $K$-[12] color such that the reddest stars $(K-[12]>3 \mathrm{mag})$ exhibit a wide range in $2-0$ bandhead depth, while the least reddened have the deepest 2-0 bandheads, with a small range of variation from star to star. Gas mass loss rates for both the AGB stars and the red supergiants in our sample correlate with the $K$-[12] color, consistent with other studies. The data imply that stars with $\dot{M}_{\text {gas }}<5 \times 10^{-7} M_{\odot} \mathrm{y}^{-1}$ exhibit a much narrower range in the relative strengths of $\mathrm{CO} 2-0$ band features than stars with higher mass loss rates. The range in observed spectral properties implies that there are significant differences in atmospheric structure among the stars in this sample.
\end{abstract}

Key words. stars: AGB and post-AGB - stars: atmospheres - stars: supergiants - infrared: stars

\section{Introduction}

Cool giants and supergiants typically experience phases of mass loss, sometimes at very high rates, which may strongly affect the evolution of the star. In the case of asymptotic giant branch (AGB) stars, the rate of mass loss exceeds the rate of core growth, so that mass loss is the most important process controlling the evolution of these stars from the AGB to the planetary nebula phase. For M-supergiants, mass loss rates up to $\sim 10^{-4} M_{\odot} \mathrm{y}^{-1}$ are inferred for timescales of order $10^{5}$ years, which implies that the evolutionary consequences may be substantial also for these massive stars $\left(M>10 M_{\odot}\right)$.

The physical mechanisms which drive mass loss are not yet well-understood, though there has been progress very recently with detailed hydrodynamic calculations of models for pulsating AGB stars (Fleischer et al. 1992; Bessell et al. 1996; Winters et al. 1997, 2000; Höfner et al. 1998; Loidl et al. 1999). These models show that the structure of the atmospheres in large-amplitude pulsators is

\footnotetext{
Send offprint requests to: J. Bieging,

e-mail: jbieging@as.arizona.edu

* Figures 4-9, 11-14, 16, 17, 19-21, 23, 24 are only avalaible in electronic form at http://www.edpsciences.org
}

strongly dependent on hydrodynamic processes which include pulsation-driven shocks, non-equilibrium chemistry, and formation of dust grains. Particularly important are the model predictions for time-dependent structure not only over single pulsation cycles but also temporal variations predicted over multiple cycles. The atmospheric structure and its changes with time determine the rate and velocity at which matter is lost, and the amount of dust which is formed and ejected. To understand the mass loss process, then, it is crucially important to compare predictions of the hydrodynamic models with all possible observable properties of such stars to assess the validity of the models.

Comparisons between hydrodynamic model predictions and selected observational properties have been made in several recent papers. Winters et al. (1997) successfully predicted the spectral energy distribution (SED) and light curve at several wavelengths for the extreme carbon star AFGL 3068, using a self-consistent timedependent model incorporating hydrodynamics, chemistry, and dust formation. Hron et al. (1998) compared low-resolution ISO-SWS $2-15 \mu \mathrm{m}$ spectra of the carbon star R Scl at two phases with both hydrostatic and dynamic atmosphere models. They find moderately good 
agreement with the dynamic models, and note that the observed time variations require a dynamic treatment of the model atmosphere. Loidl et al. (1999) calculated synthetic spectra from 0.5 to $12 \mu \mathrm{m}$ for AGB carbon stars using an opacity sampling method, with the atmospheric structure from dynamical models. They include 7 major carbon-bearing molecules in the opacities, and explore the different time dependencies of various spectral features as a result of the different layers in which molecules form. They note that their synthetic spectra reproduce qualitatively the spectra of cool carbon stars, but that higher spectral resolution is needed in the models to compare with data from the ISO-SWS, for example.

Bessell et al. (1996) and Scholz \& Wood (2000) made hydrodynamical models of M-type Miras and calculated detailed absorption line profiles of CO 1st overtone lines (among others). They compared their model spectra with the velocity results of Hinkle et al. $(1982,1984)$ to estimate the true pulsational velocities of observed Miras. They find that the $\mathrm{CO}$ absorption line profiles exhibit complex variability not only over one pulsational cycle, but also from cycle to cycle. They note the scarcity of observational data at present to compare with their predictions.

Aringer et al. (1999) studied the $4 \mu \mathrm{m}$ first overtone ro-vibrational bands of $\mathrm{SiO}$ in oxygen-rich AGB stars, and compared their observed spectra with hydrostatic and dynamic atmosphere models. They confirm the strong variability of the $\mathrm{SiO}$ band strengths with light phase for Miras, and show that this variation is consistent with pulsationally driven hydrodynamic models. In contrast, hydrostatic models cannot explain the observed $\mathrm{SiO}$ band strengths in cool giants, a shortcoming which emphasizes the need for dynamical models.

In a high resolution study of molecular hydrogen absorption lines at $\sim 2.2 \mu \mathrm{m}$ in 30 Miras and semiregular AGB stars, Hinkle et al. (2000) find a wide range of line intensities. For a few stars, they have time-series spectra which show large changes in the $\mathrm{H}_{2} 1-0 \mathrm{~S}(1)$ line intensity over the light cycles, probably reflecting dissociation and re-formation of $\mathrm{H}_{2}$ with the passage of pulsational shocks. Such behavior is in fact predicted in the dynamical atmosphere models of Höfner (1999).

Finally, in the most detailed calculation published to date, Winters et al. (2000) compare velocity-resolved model spectra with high-resolution observations of the CO $v=1-0$ fundamental and $v=2-0$ first overtone bands in the carbon star IRC +10216 . They find that dynamical models for the atmosphere can produce line profiles which are in good agreement with the observations for reasonable model parameters. Even the temporal variations, reflecting the formation and expansion of dust shells, are in rather good agreement with the observed spectra. In other details such as mass loss rate, however, there are discrepancies which suggest that further refinements to the models and more extensive observations are needed.

The CO molecule offers several advantages as a diagnostic probe of the structure of cool stellar atmospheres. It has a stable, closed-shell structure and a high dissociation energy, so it is predicted to form readily in cool giant and supergiant atmospheres, with an abundance close to the chemical thermodynamic equilibrium (TE) value. Even if the gas is being shocked periodically by stellar pulsations, shock chemistry models predict that the CO abundance is scarcely altered from the TE value (Willacy \& Cherchneff 1998; Duari et al. 1999). CO should take up almost all available carbon or oxygen, whichever is less abundant, so the $\mathrm{CO}$ molecular abundance can be reliably estimated from atomic abundances. The CO ro-vibrational spectrum has bands in the near-IR which are not too seriously affected by telluric absorption. With modern IR detector arrays, the CO bands are accessible to sensitive spectrometers at the fundamental and overtone wavelengths. The molecular constants are well-determined so that line wavelengths and transition strengths can be calculated reliably.

The detailed spectral models for IRC +10216 by Winters et al. (2000) show that lines of the CO fundamental band at $4.6 \mu \mathrm{m}$ are optically thick at least in the core, while the first overtone lines, in particular the $v=2$ 0 band, are not optically thick, even in models with large mass loss rates and dense extended atmospheres. Since CO should form deep in the photosphere and exist essentially unchanged in abundance out to large distances above the photosphere, this predicted lack of saturation in the first overtone lines suggests that they should be very useful probes for the entire extended atmosphere. The energy level structure of the CO molecule places within a relatively small spectral window ro-vibrational lines originating from a wide range in energy levels. Specifically, the intensities of absorption lines across the $v=2-0$ band at $2.3 \mu \mathrm{m}$ probe energies ranging from the ground state to $E / k>20000 \mathrm{~K}$ within a spectral range $<0.1 \mu \mathrm{m}$.

For all these reasons, the CO first overtone bands at $2.3 \mu \mathrm{m}$ should be useful diagnostics of stellar atmospheres models for cool giants and supergiants. In this paper, we present spectra of selected AGB and post-AGB stars and late-type supergiants, from 2.28 to $2.36 \mu \mathrm{m}$. This wavelength range covers the main part of the $v=2-0$ and $3-1$ CO bands, and parts of the $v=4-2$ and the ${ }^{13} \mathrm{CO} v=$ $2-0$ bands. The spectral resolution is sufficient to separate a large number of the $R$ - and $P$-branch lines, which span a wide range in energy levels. The spectra should be of value for making comparisons with the predictions of current hydrodynamic models for cool giant and supergiant atmospheres.

\section{Observations and data reduction}

\subsection{Instrument and observing method}

The observations were made with the Steward Observatory $1.54 \mathrm{~m}$ telescope on Mt. Bigelow, Arizona (elevation $2510 \mathrm{~m}$ ) on 1993 October 5 and 6 and 1994 Sep. 12 and 13. The spectrometer was at the Cassegrain focus of the f/45 infrared-optimized chopping secondary.

The spectrometer, described by Williams et al. (1993), has a 120 arcsec long slit which illuminates a liquid 
nitrogen cooled grating. The grating used was ruled at $600 \mathrm{l} / \mathrm{mm}$ blazed for $\lambda 2.5 \mu \mathrm{m}$. The detector was a NICMOS3 $256 \times 256 \mathrm{HgCdTe}$ array. The resolution at $\lambda 2.3 \mu \mathrm{m}$ with this grating is $\Delta \lambda=0.0007 \mu \mathrm{m}$, or $R \equiv$ $\lambda / \Delta \lambda=3500$ with a slit width of 1.8 arcsec, which was used for most of the data presented. During the second observing period, a 3.6 arcsec slit was used to allow adequate guiding. (As a result the resolution was degraded to $R \approx$ 2500 for a few stars as noted below.) The grating setting used gave a wavelength range from 2.28 to $2.36 \mu \mathrm{m}$.

Observations were made by moving the telescope so as to place the star at six positions along the slit. The detector was read out for each position and the separate frames were differenced to subtract the sky and yield two independent spectra of the star. Integration times per exposure ranged from $0.5 \mathrm{~s}$ to $30 \mathrm{~s}$, depending on the stellar magnitude.

Standard stars, selected mainly from the Bright Star Catalog, were dwarfs within a few degrees of the target stars, and with spectral types between early $\mathrm{F}$ and late B. Wallace \& Hinkle (1997) have shown that dwarfs with spectral types from B3 to F5 have essentially featureless spectra in the wavelength range $2.2-2.4 \mu \mathrm{m}$ when observed at $R \approx 3000$. Standard and program stars were almost always observed in pairs at nearly identical airmasses, in an "ABBA" sequence, i.e., two pairs per observation. Integration time on the standard stars was typically $30 \mathrm{~s}$ per exposure. No absolute photometry was attempted in either observing period. Wavelength calibration was determined to first order with exposures of a NeKr lamp, and refined by an iterative procedure of line identification described below.

\subsection{Source list}

Stars were selected to provide a representative sample of $\mathrm{M}$ supergiants and AGB stars of M, S, and C spectral types. Two RV Tauri variables, a class believed to be in a postAGB evolutionary stage, were also observed. In total, we observed $8 \mathrm{M}$ supergiants, $5 \mathrm{M}$ giants, $3 \mathrm{~S}$ stars, 6 carbon stars, and 2 RV Tauri variables. Their properties are summarized in Table 1. Spectral types are from the SIMBAD database, except as noted. Variability class and period are from the General Catalog of Variable Stars (GCVS Kholopov et al. 1985). $K$-band magnitudes were from the Two Micron Sky Survey (Neugebauer \& Leighton 1969) except as noted. Most of these objects are strongly variable in the visual, but at $K$-band the variability is much less, typically $<1 \mathrm{mag}$ in total range. The gas mass loss rates in column 6 were determined from model fits to observations of the CO $J=1-0$ and/or 2-1 mm-wavelength transitions, taken from the literature as noted. For the Miras with well-determined periods, the phase of the light cycle for the observation is given in Col. 8. The period and reference date were taken from GCVS, and we follow the (somewhat inconsistent) convention used there that zero phase is at maximum visual light for the Miras, but is at the deeper minimum in the cycle for the RV Tauri variables. The standard stars, listed in Col. 9, all have spectral types consistent with a nearly featureless spectrum in the observed wavelength range at $R=3500$.

The integration time in Col. 11 is the total for all target star exposures averaged into the final spectra. The last column lists an estimate of the rms noise level of the final spectrum. Because almost the entire spectral range covered is filled with absorption features for these stars, it is not possible to calculate a noise level in a region of line-free continuum. Instead, we calculated the ratio spectrum of the difference over the sum of the two observations of a given star in the ABBA sequence, for both the standard and the target, and computed the rms values across these ratio spectra. The rms in Table 1 is then the root-sum-squared of the rms values for standard and target stars, since the final spectrum is the ratio of the target star spectrum divided by the standard spectrum, expressed in terms of the normalized flux. Typical values of the rms noise are in the range $1-3 \%$ of the continuum.

\subsection{Data reduction}

The data were reduced with the IRAF software package. Individual exposures (usually 6) were shifted and combined to produce a single two-dimensional spectrum. Flatfielding was done with a dark current-corrected dome flat. A one-dimensional spectrum was produced with the IRAF aperture extraction tasks, for both the program and standard stars. Finally, the program star spectra were divided by the corresponding standard star spectra to remove telluric features.

Most of the stars were observed at two spectrometer grating settings, offset by either 0.5 or 1.5 resolution elements. This was done to ensure that the full resolution was achieved, since the spacing of the detector elements produced just $\Delta \lambda / 2$ sampling of the spectrum. To combine the spectra taken at two grating settings, the telluriccorrected spectra of the program stars were interpolated onto a finer grid in $\lambda$, then cross-correlated to determine the exact shift in pixels between the two grating settings. One spectrum was shifted with respect to the other by regridding with the offset determined by cross-correlation, and the two spectra were summed. Finally, the summed spectrum was multiplied by a normalized $10000 \mathrm{~K}$ blackbody spectrum to correct for the slope introduced in dividing by the standard star. At $\lambda 2.3 \mu \mathrm{m}$, the difference in slope between a $7500 \mathrm{~K}$ and a $15000 \mathrm{~K}$ blackbody is negligibly small across the observed band, so a $10000 \mathrm{~K}$ spectrum, appropriate to an A0V star, was adopted.

\subsection{Wavelength calibration and line identification}

An initial estimate of the dispersion functions of the measured spectra was made from exposures of a neon-krypton lamp. These were not taken at grating settings identical to all those used for the stars, however, and the location 
Table 1. Stellar properties and log of observations.

\begin{tabular}{|c|c|c|c|c|c|c|c|c|c|c|c|}
\hline \multirow[b]{2}{*}{ Star } & \multirow{2}{*}{$\begin{array}{l}\text { Spec. } \\
\text { Type }\end{array}$} & \multicolumn{4}{|l|}{ Var. } & \multirow{2}{*}{$\begin{array}{l}\text { Obs. } \\
\text { Date }^{a}\end{array}$} & \multirow{2}{*}{$\begin{array}{l}\text { Light } \\
\text { Phase } \\
\text { (cycles) }\end{array}$} & \multicolumn{2}{|c|}{ Standard Star } & \multirow{2}{*}{$\begin{array}{l}\text { Int. } \\
\text { time } \\
\text { (s) }\end{array}$} & \multirow[b]{2}{*}{ RMS } \\
\hline & & Class & $\begin{array}{l}\text { Period } \\
\text { (d) }\end{array}$ & $\begin{array}{l}K \\
(\mathrm{mag})\end{array}$ & $\begin{array}{l}\dot{M}_{\text {gas }} \\
\left(M_{\odot} \mathrm{y}^{-1}\right)\end{array}$ & & & Name & Sp.type & & \\
\hline
\end{tabular}

(a) Supergiants

$\begin{array}{llllllllllll}\text { SU Per } & \text { M3.5Iab } & \text { SRc } & 470 & 1.50 & \ldots & 236 & - & \text { HR 870 } & \text { F7IV } & 24 & 0.037 \\ \text { S Per } & \text { M3Iae } & \text { SRc } & - & 1.31 & 1.4 \mathrm{e}-6^{f} & 236 & - & \text { HR 870 } & \text { F7IV } & 24 & 0.037 \\ \text { BI Cyg } & \text { M4 } & \text { Lc? } & - & 0.58^{b} & \ldots & 236 & - & \text { HR 7769 } & \text { A2V } & 24 & 0.034 \\ \text { KY Cyg } & \text { M3.5Ia } & \text { Lb } & - & 0.28 & \ldots & 237 & - & \text { HR 7784 } & \text { A1V } & 24 & 0.048 \\ \text { NML Cyg } & \text { M4.5-M7.9 } & \ldots & - & 0.62 & \ldots & 237 & - & \text { HR 8028 } & \text { A1V } & 24 & 0.033 \\ \mu \text { Cep } & \text { M2Iae } & \text { SRc } & - & -1.88^{b} & 9.1 \mathrm{e}-8^{f} & 236 & - & \text { HR 8357 } & \text { B6V } & 12 & (0.03) \\ \text { PZ Cas } & \text { M3Ia } & \text { SRa } & - & 0.98 & 8.3 \mathrm{e}-6^{f} & 236 & - & \text { HR 9019 } & \text { A0V } & 24 & 0.030 \\ \text { TZ Cas } & \text { M2Iab } & \text { Lc } & - & 1.95^{b} & \ldots & 236 & - & \text { HR 9019 } & \text { A0V } & 24 & 0.037\end{array}$

(b) $M$ giants

$\begin{array}{ll}\text { W And } & \text { M7:p } \\ \text { KU And } & \text { M9 } \\ \text { T Cas } & \text { M7e } \\ \text { IK Tau } & \text { M6me } \\ \text { UX Cyg } & \text { M5 } \\ \text { (c) } S \text { stars } & \end{array}$

$\begin{array}{llllllllll}\text { M } & 396.71 & 0.88 & \ldots & 236 & 0.31 & \text { HR } 670 & \text { A1V } & 24 & 0.027 \\ \text { M } & 750: & 2.52 & 9.6 \mathrm{e}-6^{g} & 638 & \ldots & \text { HR 76 } & \text { A0V } & 60 & 0.017 \\ \text { M } & 445.0 & -0.97 & 5.1 \mathrm{e}-7^{h} & 638 & 0.41 & \text { HR 96 } & \text { B9IV } & 6 & 0.016 \\ \mathrm{M} & 500: & -1.24 & 3.8 \mathrm{e}-6^{g} & 638 & \ldots & \text { HR 1137 } & \text { A0V } & 18 & 0.016 \\ \mathrm{M} & 561.24 & 1.97^{c} & 3.2 \mathrm{e}-6^{h} & 639 & 0.56 & \text { SAO 70289 } & \text { F0V } & 36 & 0.012\end{array}$

$\begin{array}{ll}\text { R And } & \text { S6.6 } \\ \text { W Aql } & \text { S4.9 } \\ \text { AD Cyg } & \text { S5.8 }\end{array}$

(d) Carbon stars

\begin{tabular}{|c|c|c|c|c|c|c|c|c|c|c|c|}
\hline HV Cas & CVIIe+ & M & 527 & 2.32 & $\ldots$ & 236 & 0.1 & HR 343 & $\mathrm{~A} 7 \mathrm{~V}$ & 24 & 0.038 \\
\hline V466 Per & No. V & $\mathrm{SR}$ & - & 1.01 & $\ldots$ & 236 & - & HR 1160 & B8V & 24 & 0.025 \\
\hline TT Tau & C5II & $\mathrm{SRb}$ & 166.5 & 1.05 & $\ldots$ & 237 & - & HR 1554 & F2IV & 24 & 0.053 \\
\hline V Cyg & $\mathrm{C} 7.4 \mathrm{e}$ & M & 421.27 & 0.82 & $2.6 \mathrm{e}-6^{h}$ & 236 & 0.34 & HR 7958 & A3V & 24 & 0.032 \\
\hline V460 Cyg & C6.3 & $\mathrm{SRb}^{j}$ & - & 0.23 & $1.1 \mathrm{e}-6^{h}$ & 237 & - & HR 8307 & $\mathrm{~A} 0 \mathrm{~V}$ & 24 & 0.039 \\
\hline RV Cyg & C6.4II & $\mathrm{SRb}$ & 300: & 0.36 & $1.7 \mathrm{e}-6^{h}$ & 237 & - & HR 8307 & $\mathrm{~A} 0 \mathrm{~V}$ & 24 & 0.034 \\
\hline
\end{tabular}

(e) $R V$ Tauri variables

\begin{tabular}{llllllllllll} 
RV Tau & G2 Iae $^{d}$ & RVb & 78.698 & $5.0^{e}$ & $\ldots$ & 638 & 0.55 & HR 1554 & F2IV & 360 & 0.007 \\
AC Her & F2Ibp $^{d}$ & RVa & 75.4619 & $5.5^{e}$ & $\ldots$ & 639 & 0.30 & SAO 103879 & A0V & 360 & 0.009 \\
\hline
\end{tabular}

${ }^{a}$ JD2449000+; ${ }^{b}$ Josselin et al. (2000); ${ }^{c}$ Feast \& Whitelock (2000); ${ }^{d}$ Oudmaijer et al. (1995); ${ }^{e}$ Gezari et al. (1999); ${ }^{f}$ Josselin et al. (2000); ${ }^{g}$ Neri et al. (1998); ${ }^{h}$ Loup et al. (1993); ${ }^{i}$ Bieging \& Latter (1994); ${ }^{j}$ Jørgensen et al. (2000).

of the star on the slit introduced a zero-point offset in the dispersion function, so it was necessary to use an iterative line identification procedure to determine an accurate wavelength calibration for each target star spectrum. As noted in the introduction, the $\mathrm{CO}$ rovibrational bands are potentially useful diagnostic probes of cool stellar atmospheres because the lines arise from a very wide range of energy levels but are confined to a relatively small range in wavelength. The small spectral range means, however, that many lines are blended or even almost perfectly coincide, especially for wavelengths longward of the CO 3-1 bandhead. These properties are illustrated in Fig. 1, which shows the lower state energy of the CO $2-0,3-1$, and
4-2 bands and the ${ }^{13} \mathrm{CO} 2-0$ band, over the range of wavelengths covered in the spectra presented here. Wavelengths and energy levels were calculated from the molecular data of Farrenq et al. (1991). Shortward of the 3-1 bandhead, the lines are resolved at $\Delta \lambda=0.0007 \mu \mathrm{m}$ up to about R30. Longward of the 3-1 bandhead, the lower $R$ - and $P$-branch lines of the $2-0$ band are in some cases blended and in others just resolved from the $R$-branch lines of the 3-1 band.

These spectral characteristics of the CO bands led us to an iterative procedure to refine the wavelength calibration and to identify as many spectral features as possible. First, the 2-0 and 3-1 bandheads are usually readily 


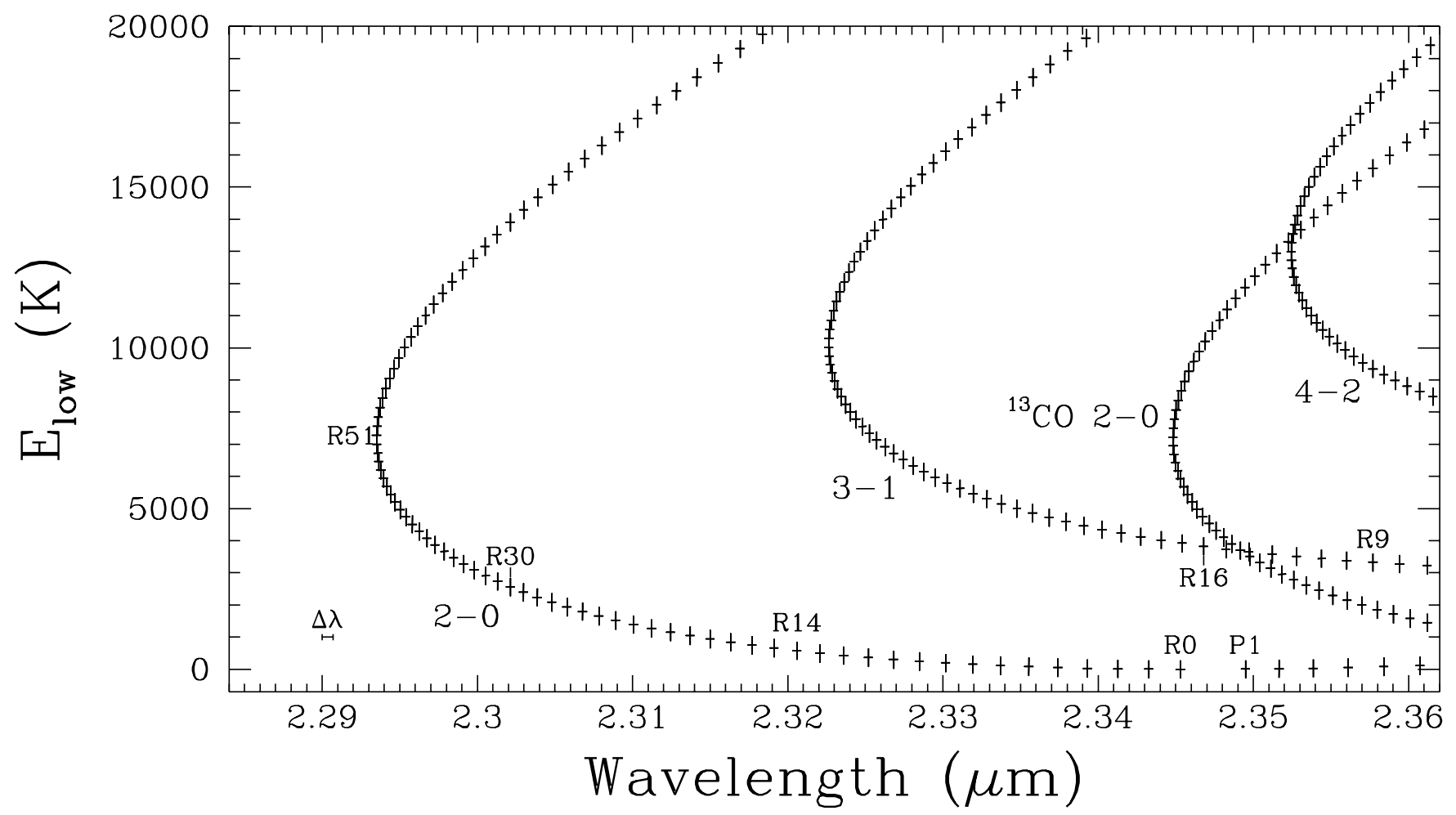

Fig. 1. Energy levels of CO 1st-overtone bands in the wavelength range observed. Lower-state energies expressed in temperature units, $E / k$, are plotted versus wavelength for the $2-0,3-1$, and $4-2$ bands of ${ }^{12} \mathrm{C}^{16} \mathrm{O}$ and for the $2-0$ band of ${ }^{13} \mathrm{C}^{16} \mathrm{O}$. Selected transitions are labelled. Horizontal bar at lower left shows resolution, $\Delta \lambda$, of the spectra.

identified. The 3-1 head, however, is blended with the $2-$ $0 R 12$ line, but the $R 14$ line lies shortward of the $3-1$ head just enough to be well resolved at $R=3500$. Higher $R$-branch lines up to about $R 31$ are well-separated, so we used the $2-0 R 14$ and $R 26$ lines, and the $2-0$ bandhead wavelengths. On the longward side, the $2-0 R 0$ line, blended with the ${ }^{13} \mathrm{CO} 2-0$ bandhead was always recognizable and usually the 4-2 bandhead (but blended with $3-1$ R12). These identifications were used to derive a dispersion function from a 3-segment linear spline fit with IRAF. Because the bandheads are blended and have shapes which should depend on the properties of the stellar atmospheres, we made a second iteration using only lines that were unblended at $\Delta \lambda=0.0007 \mu \mathrm{m}$, or that were coincident with another line. The second pass lines included $2-0$ band $R 6, R 7, R 8$, and $R 14$ through $R 31 ; 3-1$ band $R 15, R 16$, and $R 21 ; 2-0$ P4 blended with $3-1 R 10$; and the 2-0 bandhead. Typical scatter about the fitted dispersion function was $<0.0002 \mu \mathrm{m}$ peak-to-peak or an rms scatter of $<10 \%$ of a resolution element.

Some of the atomic lines which Hinkle et al. (1995) detected in their high resolution spectrum of Arcturus are also evident in our spectra. These lines are heavily blended with CO lines except at the edge of the spectra shortward of the 2-0 bandhead, where the noise level increases, so no atomic lines were used in the fits to determine the dispersion functions.

\section{Spectra}

\subsection{Identified lines}

The final spectra are shown in Figs. 2-251, grouped by stellar category. The flux scale is normalized to unity at the peak at $\lambda 2.293 \mu \mathrm{m}$, just shortward of the $v=2-0$ bandhead. The estimated $1-\sigma$ noise level is shown by a vertical error bar on the left side. The resolution is $\Delta \lambda=$ $0.0007 \mu \mathrm{m}$, or $R=3500$. At this resolution, the spectra exhibit a rich complexity of absorption features which are predominantly due to the $v=2-0$ and $3-1$ rovibrational lines of ${ }^{12} \mathrm{C}^{16} \mathrm{O}$, over the wavelength range covered. To aid in identifying individual features in these $\mathrm{CO}$ bands, thin vertical lines mark the wavelengths of the $P 1-P 4$ and $R 0$ $R 40$ lines of the $v=2-0$ band, and $R 10-R 40$ of the $v=3-1$ band. Also shown as thicker vertical lines are the heads of the $v=2-0,3-1$, and $4-2$ bands, and the ${ }^{13} \mathrm{C}^{16} \mathrm{O} v=$ 2-0 band. CO line wavelengths were calculated from the molecular constants in Farrenq et al. (1991). Atomic transitions which were detected in the high-resolution spectrum of Arcturus by Hinkle et al. (1995) are marked with lines labelled above the spectrum.

${ }^{1}$ Figures 2, 3, 10, 15, 18, 22, and 25 are displayed in the printed version of this paper as representative examples of the $\mathrm{M}$ supergiants, and the M, S, and C-type giants; all spectra are available in the electronic version. 


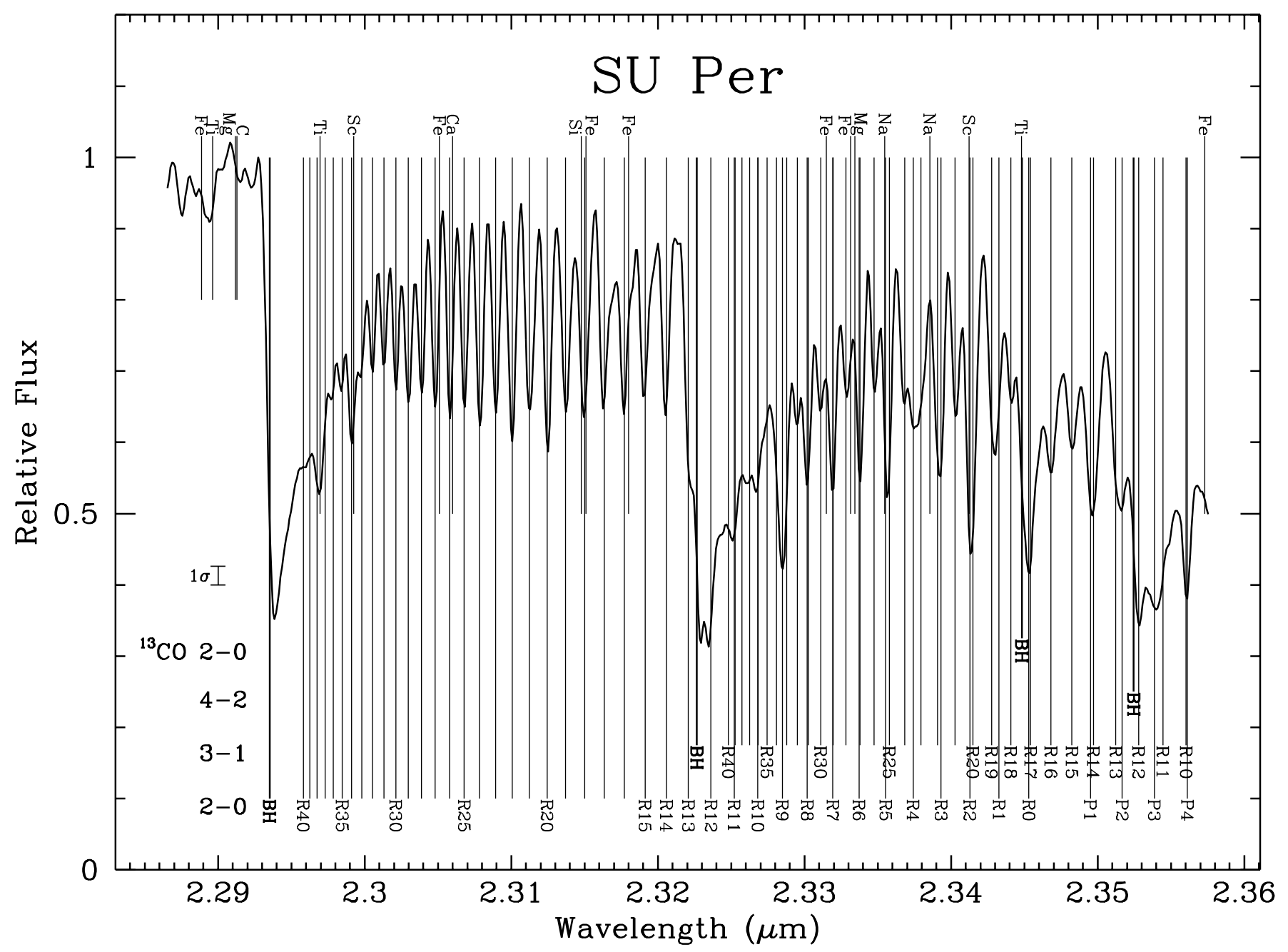

Fig. 2. Spectrum of SU Per (spectral type M3.5Iab, variability class SRc). CO lines up to $R 40$ are identified at the bottom, with bandheads as thick vertical lines. Atomic lines from Hinkle et al. (1995) are indicated at the top.

Despite the apparent complexity in Figs. 2-25, close examination of each spectrum shows that almost every feature can be identified with either a single CO line or a blend of lines. The lines from $R 14$ to about $R 35$ in the 2- 0 band are clearly resolved, and the $R 13$ line is apparent as an inflection just shortward of the $v=3-1$ bandhead. Longward of the 3-1 bandhead, the $R$-branch lines of the 2-0 and 3-1 bands are alternately blended and resolved. For example, the 2-0 $R 9$ line blends with the $3-1 R 33$ and $R 34$ lines, and 2-0 $R 7$ with 3-1 $R 20$, producing at this resolution characteristically deep, broadened absorption features. In contrast, the gap between the 2-0 $R 0$ and $P 1$ lines allows for a clear separation of the 3-1 $R 15$ and $R 16$ lines. It is quite possible that some weak absorption is present from lines at energies higher than the bandheads, i.e., $>$ R51. Hinkle et al. (1995) detected up to the 2-0 R99 line in the spectrum of Arcturus, which however has an effective temperature of $4320 \mathrm{~K}$, significantly higher than the stars in our sample. The higher energy lines may contribute weakly but the dominant $\mathrm{CO}$ absorption features are those identified in the figures.
Only a few atomic lines are readily discernible in the spectra. The most obvious are a line of Ti I at $\lambda 2.29696$ $\mu \mathrm{m}$ and a line of Sc I at $\lambda 2.29926 \mu \mathrm{m}$, both of which show up as absorption features on the flank of the $v=$ 2-0 bandhead. A few weak atomic lines are present at the short wavelength edge of the spectra, but other atomic lines, if present, are badly blended with CO lines.

\subsection{Intensities of selected features}

The spectra in Figs. $2-25$ show broadly similar structure in the $\mathrm{CO}$ bands, but there are significant differences from star to star, in the shape and depth of many features. To facilitate comparisons and search for correlations with stellar properties, we list in Table 2 the intensities of selected features, expressed in per cent of the continuum, as measured directly from the spectra. Features tabulated include the following:

(a) $v=2-0$ bandhead. The short wavelength limit is at $R 51$ but within one resolution element are blended 


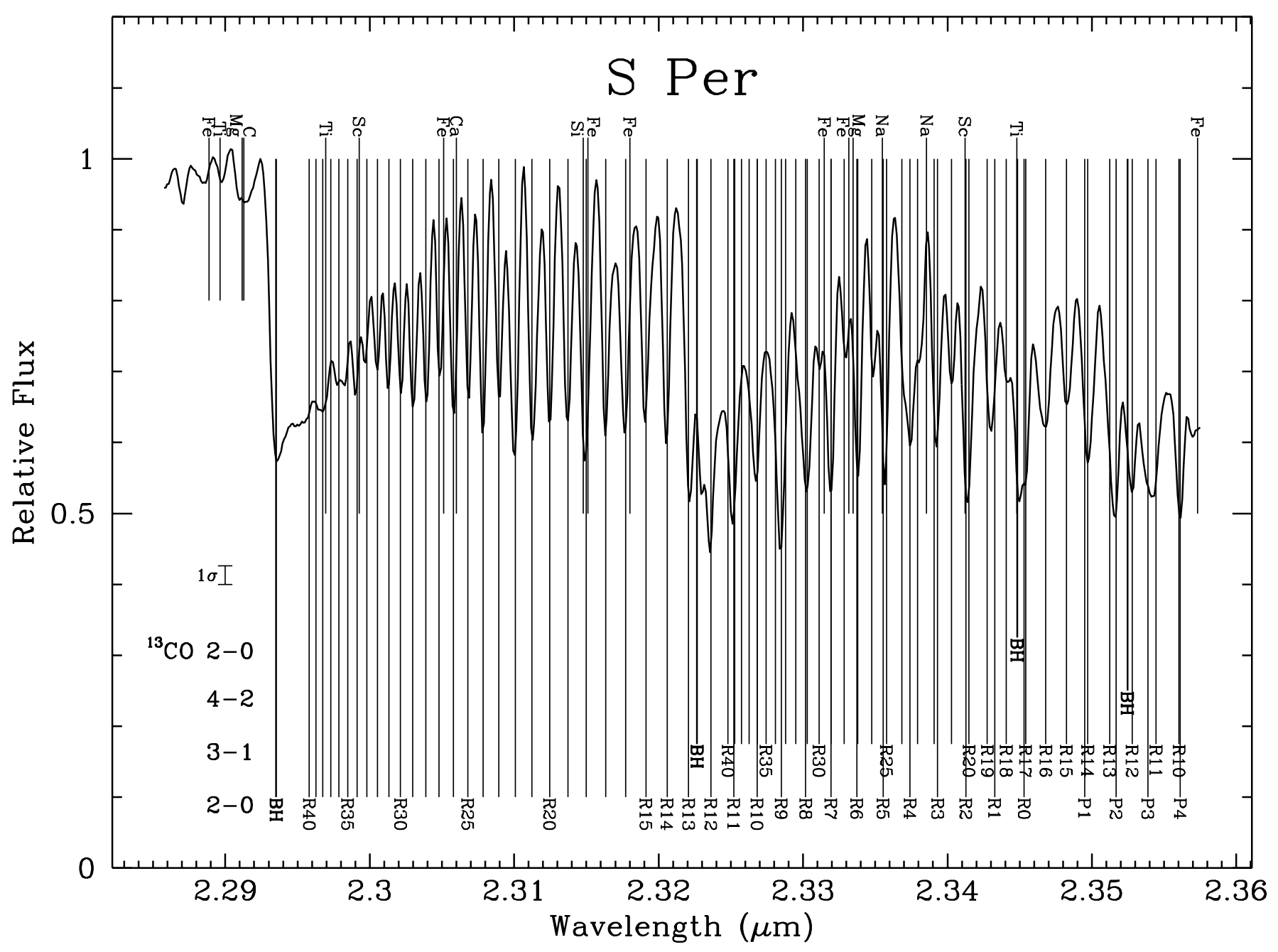

Fig. 3. Spectrum of S Per (M3Iab, SRc).

the $R 45-R 56$ lines, which span lower state energies, $E_{\text {low }}$, from 5700 to $8700 \mathrm{~K}$.

- (b) $v=2-0 R 30$. This transition lies at $E_{\text {low }}=2565 \mathrm{~K}$, but is nearly coincident in wavelength with the R71 line at $E_{\text {low }}=13911 \mathrm{~K}$.

- (c) $v=2-0 R 14$. This line has $E_{\text {low }}=581 \mathrm{~K}$. Its wavelength lies between, but is just resolved from, $R 86$ and $R 87$.

- (d) $v=2-0 R 0$. Though one of the more prominent features in most of our spectra, this ground-state line is blended with both the $v=3-1 R 17$ line $\left(E_{\text {low }}=\right.$ $3922 \mathrm{~K})$ and the ${ }^{13} \mathrm{CO} v=2-0$ bandhead $\left(E_{\text {low }} \approx\right.$ $7300 \mathrm{~K})$.

- (e) $v=3-1$ bandhead. The shape of the bandhead is influenced by the proximity of the $2-0 R 12$ and $R 13$ lines (with $E_{\text {low }}=431 \mathrm{~K}$ and $503 \mathrm{~K}$ respectively). The bandhead lines have $E_{\text {low }} \approx 10000 \mathrm{~K}$.

- (f) $v=3-1 R 16$ and $R 15$. These lines arise from levels with $E_{\text {low }} \approx 3800 \mathrm{~K}$. They fall between the $2-0 R 0$ and $P 1$ lines, but are blended with ${ }^{13} \mathrm{CO} v=2-0$ lines near that bandhead. (g) $v=4-2$ bandhead. Lines near the bandhead have $E_{\text {low }} \approx 13000 \mathrm{~K}$, but are blended with the $3-1 R 12$ line $\left(E_{\text {low }}=3511 \mathrm{~K}\right)$.

\subsection{Comparison with other spectra}

The most directly comparable published spectra for any stars in our sample are those of Kleinmann \& Hall (1986) and Wallace \& Hinkle (1997), who present spectra for 4 stars in common: $\mu$ Cep, SU Per, KY Cyg, and PZ Cas - all M supergiants. (Kleinmann \& Hall included $\mu$ Cep and SU Per in their atlas and in fact the same observational data were processed by Wallace \& Hinkle 1997.) We have compared our spectra with those of Wallace \& Hinkle (1997) and find good agreement, considering the difference in effective resolution. Wallace \& Hinkle used the Fourier Transform Spectrometer (FTS) on the NOAO 4-m telescope and presented spectra with an effective frequency resolution of $1.6 \mathrm{~cm}^{-1}$ after processing, corresponding to $R=2700$ or $\Delta \lambda=0.00086 \mu \mathrm{m}$ at $\lambda 2.32 \mu \mathrm{m}$. At this somewhat lower resolution than ours $(R=3500)$, the CO bandheads show virtually identical intensities and shapes 


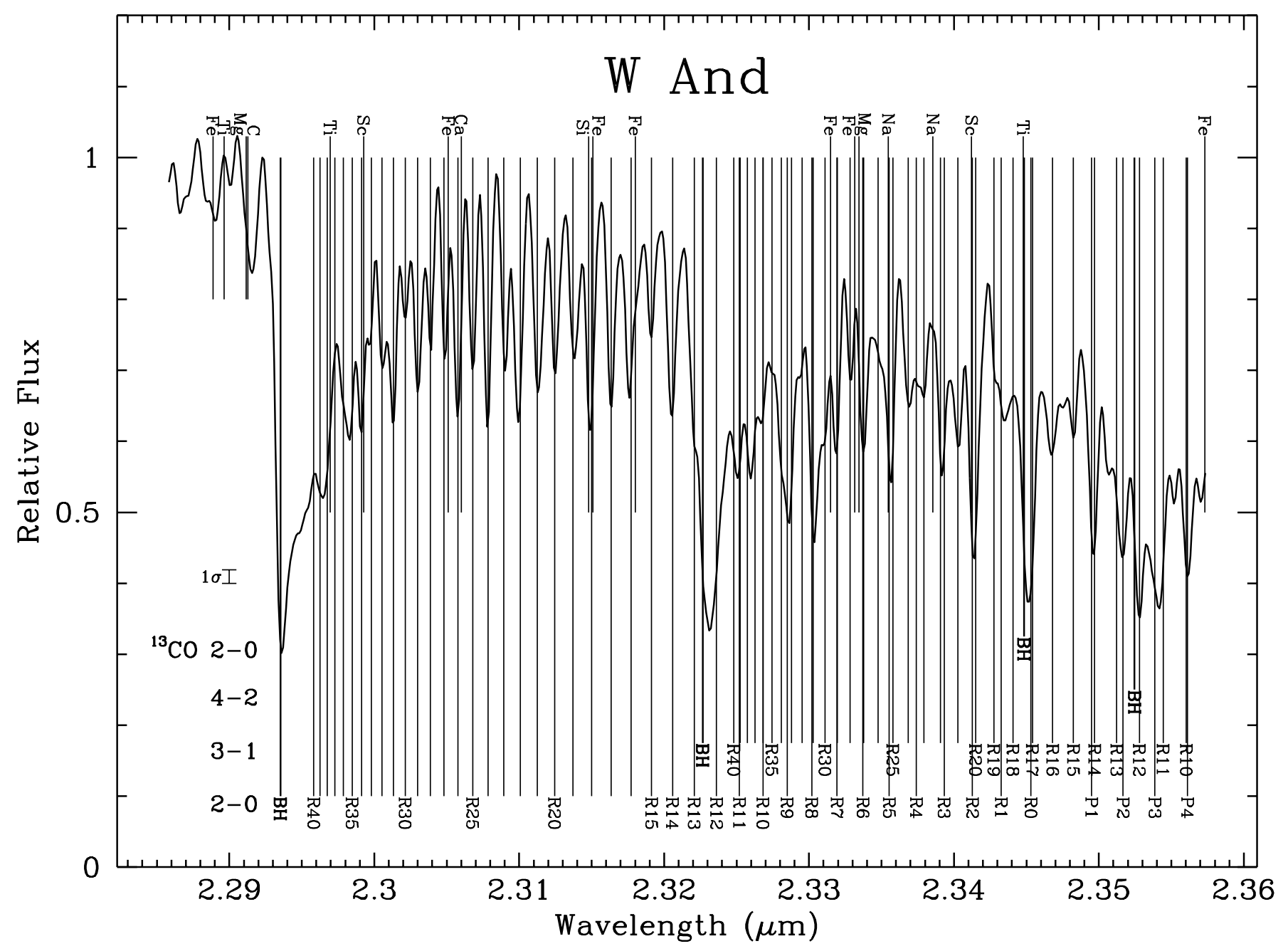

Fig. 10. Spectrum of W And (M7:p, Mira).

but the individual rovibrational lines (e.g., 2-0 R14-R30) are less well-resolved, as expected. An example which compares spectra for $\mu$ Cep is shown in Fig. 26. These observations were separated in time by over 12 years, so spectral variability is a possibility, but the main differences can be explained simply by the difference in effective resolution.

\section{Discussion}

\subsection{Correlations among spectral features}

Kleinmann \& Hall (1986) noted that the CO 2-0 and 3-1 bandhead intensities were highly correlated for the stars in their atlas. We find the same correlation in our data, as can be seen in Table 2 . The 3-1 bandhead at our resolution is somewhat blended with the $2-0 R 12$ and $R 13$ lines, which must affect the measured intensity of the 3-1 bandhead. The $2-0$ and $3-1$ bandheads have nearly equal absorption depths in all our stars, spanning a range from $27 \%$ to $93 \%$ of the continuum. The mean ratio of $I(3-1 B H) / I(2-0 B H)$ for all stars in Table 2 is $0.95 \pm 0.08$, where $I(F)$ is the normalized intensity of feature $F$ from Table 2. There is no significant difference between stellar categories. Individual transitions in the $v=2-0$ band and shortward of the $3-1$ head also show strong correlations - e.g., the $R 14$ and $R 30$ line intensities have a mean ratio $I(R 30) / I(R 14)=1.04 \pm 0.06$, with no difference between categories. (Note that the $R 30$ line coincides with the $R 71$ line.) The intensity of the $2-0 R 0$ feature is less well-correlated with that of $R 14$, but the $R 0$ line is blended with the $3-1 R 17$ line and the ${ }^{13} \mathrm{CO} 2-0$ bandhead, so a larger scatter is not surprising.

A more interesting relation is found in comparing the intensity of the $2-0 R 14$ line with the $2-0$ bandhead, as shown in Fig. 27. There appears to be a kind of saturation effect in that as the 2-0 bandhead gets deeper $(I(2-0 B H)<40 \%)$, the $R 14$ line approaches a limiting value of $\sim 60 \%$ of the continuum. The trend in Fig. 27 seems well-defined for the AGB stars, but the red supergiants generally lie below or close to the AGB stars. The 9 Mira variables in our sample are indicated by circles in Figs. 27-33. In Fig. 27, the Miras show a tight linear correlation, but the non-Mira AGB stars follow the same trend with no more scatter than the Miras. For the plotted quantities, then, there seems to be no difference between variability classes for the AGB stars. 


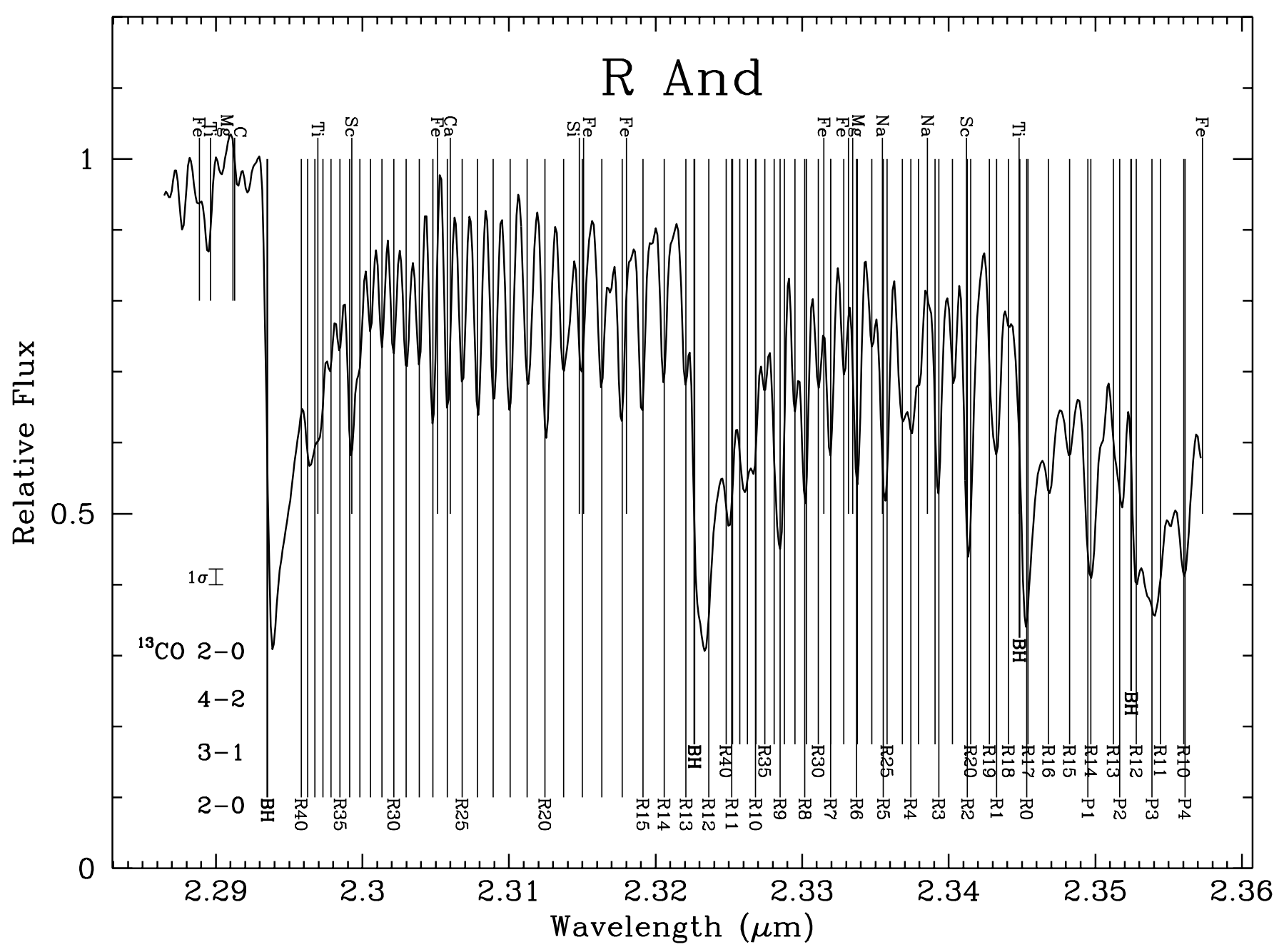

Fig. 15. Spectrum of R And (S6.6, Mira).

An alternative way to compare these features is to plot the ratio of the absorption depths vs. $I(2-0 B H)$, where the absorption depth (in per cent) is defined as $100-I(F)$. Figure 28 shows the ratio of the absorption depth of $R 14$ over the 2-0 bandhead. For the stars with the deepest 2-0 bandheads $(I<40 \%)$, the depths of the $R 14$ line all cluster near 0.5 of the depth of the $2-0$ head. The ratio appears to show an increasing scatter for shallower 2-0 bandheads ( $>40 \%$ ). Taking the AGB stars and the red supergiants separately, however, Fig. 28 suggests that there are two different correlations present-one for the giants and another for the red supergiants. The Miras and non-Mira AGB stars in Fig. 28 are not distinguishable in the average trend nor the scatter about it. The two RV Tauri variables do not obviously fit with either trend.

\subsection{Correlations with IR colors}

The infrared color $K$-[12] should be a measure of the dust opacity of the circumstellar envelope for cool masslosing stars. Whitelock et al. (1994) inferred a relation between $\dot{M}_{\text {dust }}$ and $K$-[12] colors for AGB stars;
Josselin et al. (2000) found a similar relation for M supergiants. LeBertre (1997) and LeBertre \& Winters (1998) derived relations between the gas mass loss rates and IR colors for carbon stars and oxygen-rich (i.e., M-type) Miras, respectively. Their mass loss rates were determined from radiative transfer models for the $1-100 \mu \mathrm{m}$ spectra, and assumptions about the dust properties.

We have calculated the $K$-[12] colors for our sample using the IRAS PSC $12 \mu \mathrm{m}$ fluxes, $S_{12}$, and [12] $=-2.5$ $\log \left(S_{12} / 28.3 \mathrm{Jy}\right)$ (see the IRAS Explanatory Supplement Beichman et al. 1985). In Fig. 29, we show the gas mass loss rates for the 13 AGB stars and supergiants in our sample with published $\dot{M}_{\text {gas }}$ values (see Table 1), plotted against $K$-[12] color. There is indeed a fairly good correlation as would be expected if the dust mass loss rates determine $K$-[12] and the gas-to-dust ratios are similar for these stars. The scatter at a given $K$-[12] color does suggest a factor $\sim 5$ spread in $\dot{M}_{\text {gas }}$, which is comparable to the spread in gas-to-dust ratios inferred for samples both of supergiants (Josselin et al. 2000) and of AGB stars (Whitelock et al. 1994). If we consider only the 7 Miras for which CO-derived gas mass loss rates are available 


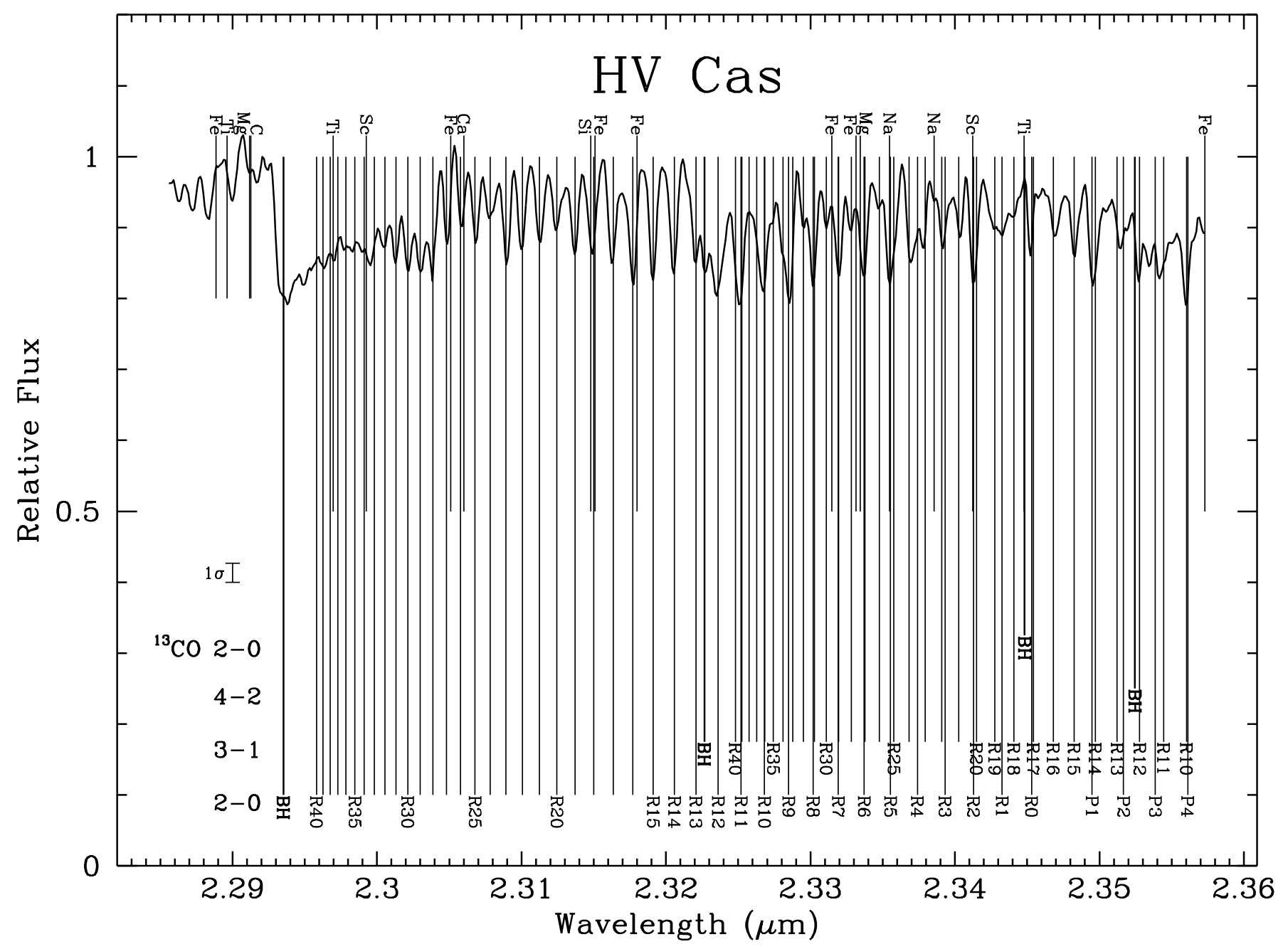

Fig. 18. Spectrum of HV Cas (CVIIe+, Mira).

(circled symbols in Fig. 29), the data points all lie on a straight line, but with a shallower slope than the relation of Whitelock et al. (1994). The trend for all the Miras in Fig. 29 is actually nearly parallel to the curve of LeBertre \& Winters (1998) for carbon stars, even though 4 of the 7 points are M-giants and 2 are S stars. Since there is only 1 carbon star in this small sample, we cannot draw any conclusions about differences between M-giants and carbon stars in the $\dot{M}-(K$-[12]) relation. Figure 29 does suggest, however, that the M-giants follow a trend which is significantly shallower than predicted by LeBertre \& Winters (1998) for their O-rich dust models.

If the $K-[12]$ colors are indicators of the gas mass loss rate, then the $\mathrm{CO}$ band features might be expected to correlate with the infrared colors as well. In Fig. 30, we plot the intensity of the $(2-0)$ bandhead against $K$-[12] color for all the stars in our sample. Clearly there is no tight correlation between the two quantities. There is, however, a trend in the data that the observed range in $(2-0)$ bandhead intensities depends on the $K$-[12] color. At the reddest colors, $>3 \mathrm{mag}$, the $(2-0)$ bandhead ranges from $30 \%$ to $>80 \%$ of the continuum. For less reddened stars
$(K-[12]<3 \mathrm{mag})$, the observed range is reduced to $27 \%$ to $52 \%$. Conversely, the deepest $(2-0)$ bandhead values $(<40 \%$ of the continuuum) correspond to the widest range in $K$-[12] colors, from 0.2 to $5 \mathrm{mag}$, while the shallowest (2-0) bandheads $(>60 \%)$ have colors in the range $3-6$ mag. If the $K$-[12] color is indeed an indicator of mass loss rate for AGB and RSG stars (but not the RV Tauri stars), as Fig. 29 suggests, then the distribution of points in Fig. 30 implies that stars with the higher mass loss rates exhibit a wider range of CO bandhead absorption than stars with lower mass loss rates. Lower rates correspond to the deepest CO absorption, with (2-0) bandheads $25 \%-50 \%$ of the continuum.

A similar trend is found if we compare the ratio of the 2-0 $R 14$ and 2-0 bandhead absorption depths as a function of $K$-[12] color. Figure 31 shows that for stars with $K$-[12] $<3 \mathrm{mag}$, the $R 14$ line depth is between 0.43 and 0.57 of the $2-0$ bandhead depth. For stars with $K$ [12] $>3 \mathrm{mag}$, the ratio spans 0.45 to 0.92 , i.e., about a 3 times larger range. If we interpret the $K$-[12] colors in terms of mass loss rates, 3 mag corresponds to $\dot{M}_{\text {gas }} \approx$ $5 \times 10^{-7} M_{\odot} \mathrm{y}^{-1}$. The data imply that stars with mass loss 


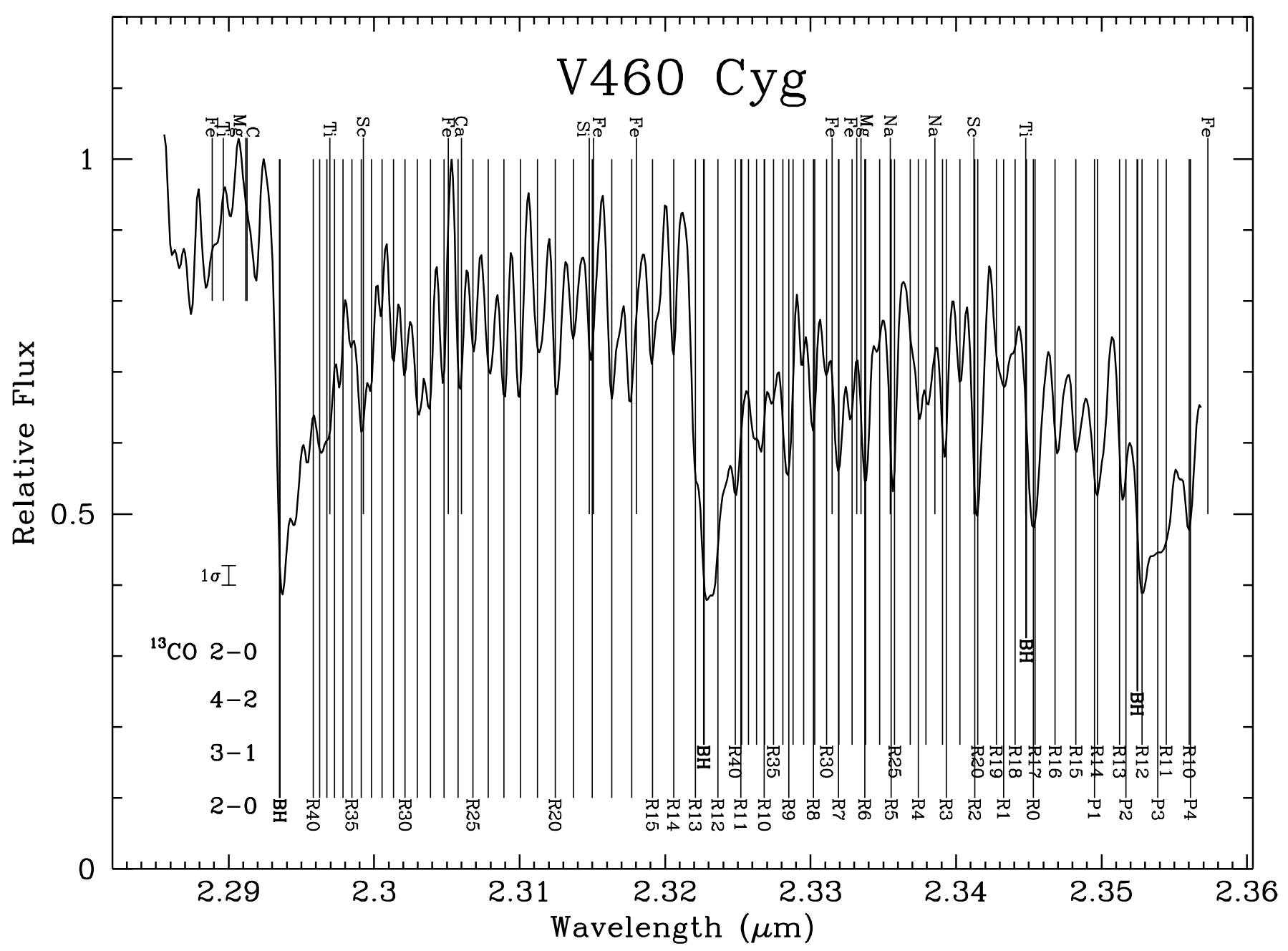

Fig. 22. Spectrum of V460 Cyg (C6.3, SRb).

rates lower than this value show a much narrower range in relative strengths of the $\mathrm{CO}$ features than do stars with $\dot{M}_{\text {gas }}>5 \times 10^{-7} M_{\odot} \mathrm{y}^{-1}$.

An alternative comparison of the $\mathrm{CO}$ features with IR color is to plot the ratio of the intensity of the $2-0 R 14$ line to the intensity of the $2-0$ bandhead, vs. $K$-[12], shown in Fig. 32. An important question in evaluating the spectra is whether the CO absorption features are being filled in by a dust continuum. If this effect were significant, the intensity ratios of features such as $[2-0 R 14 / 2-0 B H]$ should approach unity as the degree of "veiling" increases. Figure 32 shows a marginal trend in this direction, in that for $K-[12]>3 \mathrm{mag}$, the mean ratio is lower than for stars with $K$-[12] $<3$ mag. The correlation is weak, however, and at any given $K-[12]$ color, there is a significant spread in the $[R 14 / 2-0 B H]$ intensity ratios. This spread in the ratios suggests that dust continuum is probably not a major factor in determining the relative intensities of the $\mathrm{CO}$ absorption features.

Figure 33 makes a direct comparison between the 2-0 bandhead and the CO-derived gas mass loss rates for the 13 stars with $\dot{M}_{\text {gas }}$ in Table 1 . The trends are very similar to those in Fig. 30, i.e., that stars with the deepest CO bandheads $(I<40 \%)$ span a wide range in mass loss rates, while the shallower bandheads $(I>60 \%)$ are limited to the highest mass loss rates. This similarity in the trends between Figs. 30 and 33 is to be expected, given the rather good correlation between $K$-[12] and $\dot{M}_{\text {gas }}$ seen in Fig. 29.

The Mira variables in Figs. 30-33 (circled symbols) show the trend noted above. For the reddest stars $(K-$ $[12]>3 \mathrm{mag}$ ), the ranges in CO line intensities and ratios are very large, while the less reddened Miras have more restricted values of CO line properties. Since the $K$-[12] color correlates well with $\dot{M}$ for the Miras (see Fig. 29), this result implies that Miras with $\dot{M}>(5-10) \times 10^{-7} M_{\odot} \mathrm{y}^{-1}$ exhibit a larger range in atmospheric structure than do the Miras with lower $\dot{M}$. This dichotomy may possibly be related to effects found by Winters et al. (2000) in their dust-driven hydrodynamic models. At high mass loss rates $\left(>3 \times 10^{-7} M_{\odot} \mathrm{y}^{-1}\right)$, they find a large range in variation of the envelope structure with time - e.g., cycle-tocycle and multiperiodic behavior. In contrast, for lower mass loss rates, the envelopes are nearly stationary with time, so that more uniform atmospheric absorption line properties would be expected. In this theoretical context, then, the large range of CO properties at high $\dot{M}$ or large 


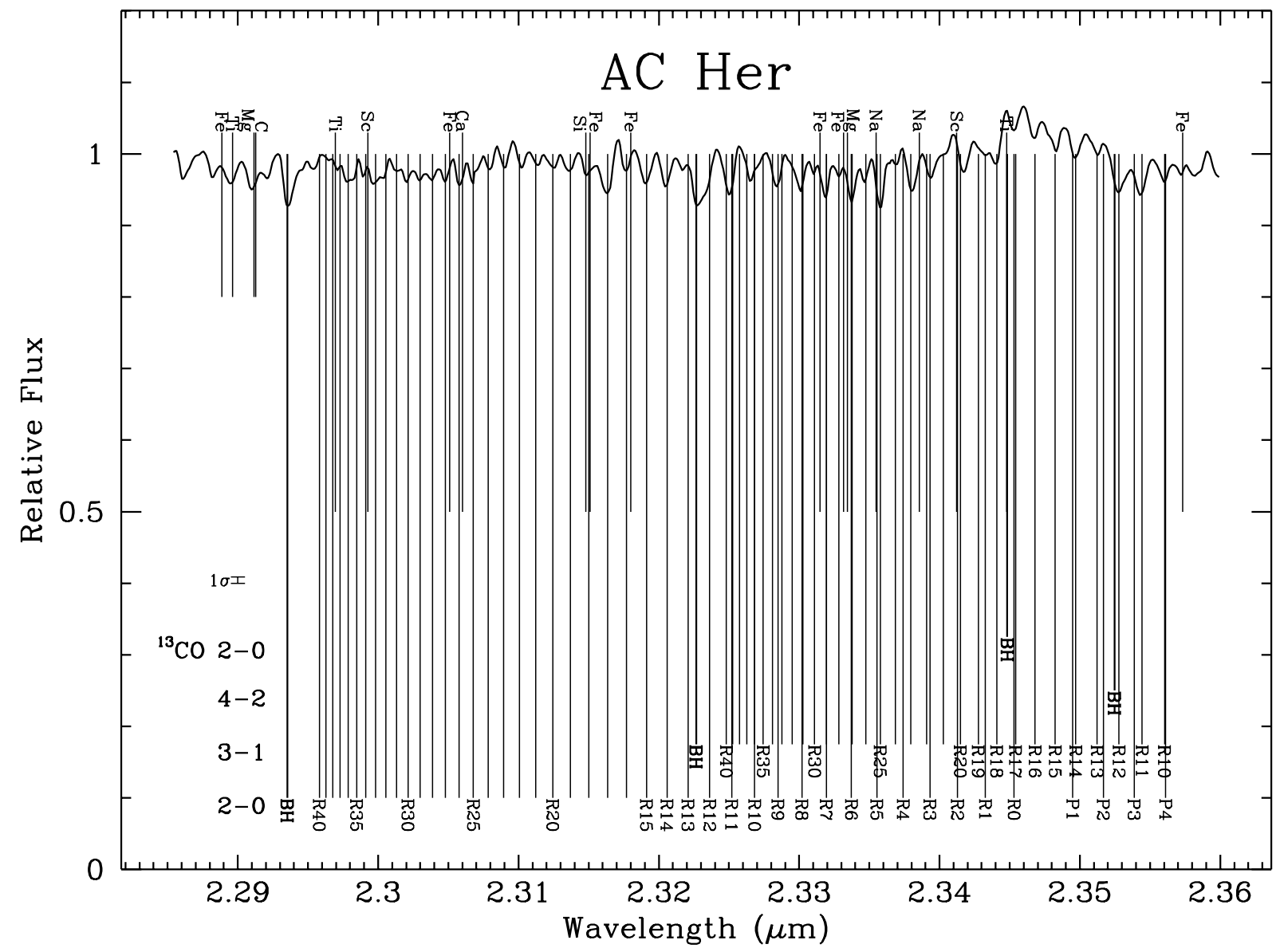

Fig. 25. Spectrum of AC Her (F2Ibp, RV Tauri variable).

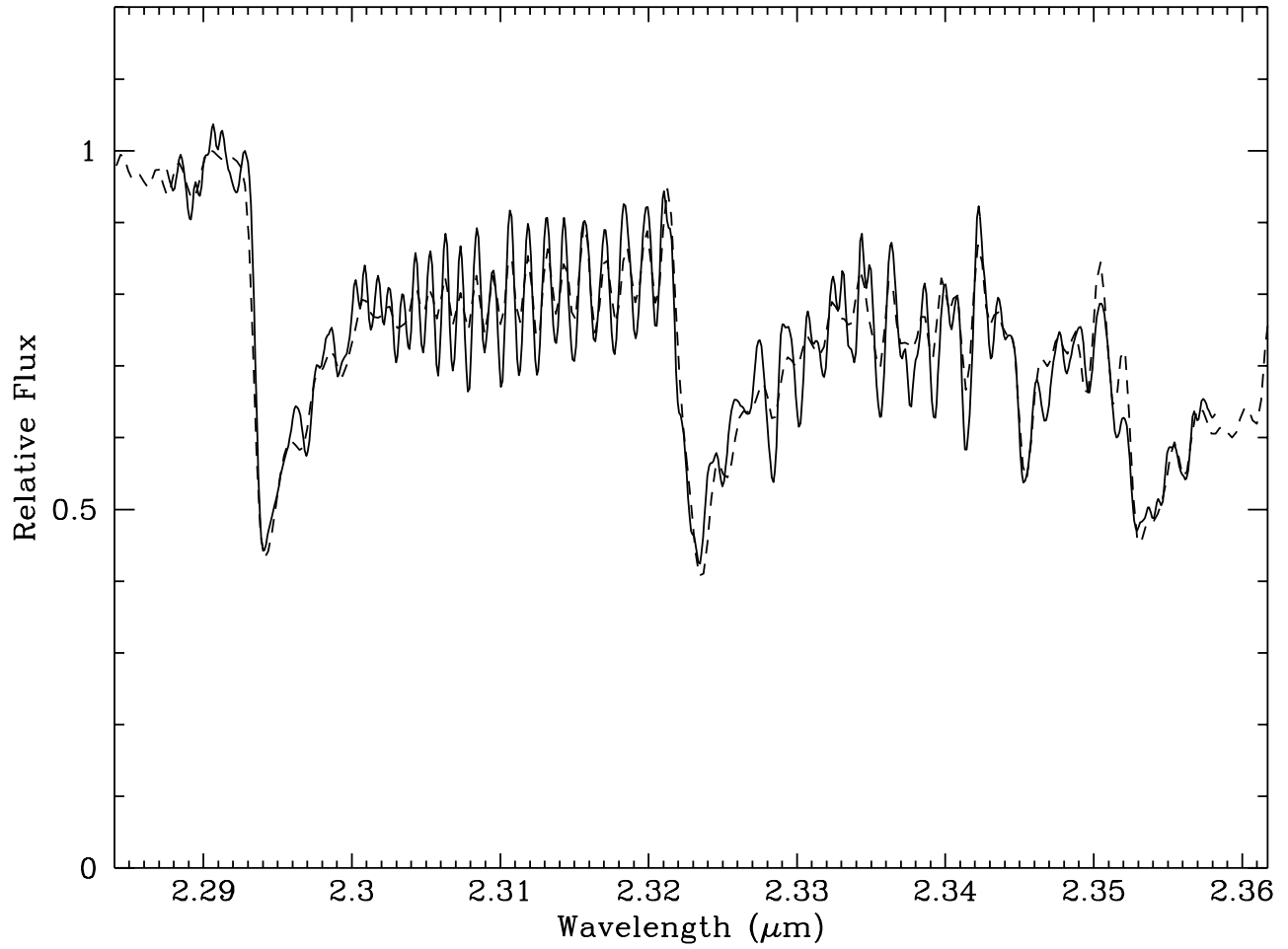

Fig. 26. Comparison of spectrum of $\mu$ Cep from this paper (resolution $R=3500$ ) (solid line) with that of Wallace \& Hinkle (1997) (resolution $R=2700$ ) normalized at the same wavelength (dashed line). 
Table 2. CO rovibrational absorption feature intensities (\% of continuum).

\begin{tabular}{|c|c|c|c|c|c|c|c|c|}
\hline \multirow[b]{2}{*}{ Star } & \multicolumn{4}{|c|}{$v=2-0$} & \multicolumn{3}{|c|}{$v=3-1$} & \multirow{2}{*}{$\begin{array}{l}v=4-2 \\
B H^{d}\end{array}$} \\
\hline & $B H$ & $R 30^{a}$ & $R 14$ & $R 0^{b}$ & $B H^{c}$ & $R 16$ & $R 15$ & \\
\hline \multicolumn{9}{|c|}{ (a) Supergiants } \\
\hline SU Per & 35 & 68 & 64 & 42 & 32 & 56 & 59 & 35 \\
\hline S Per & 57 & 67 & 61 & 54 & 45 & 62 & 66 & 53 \\
\hline BI Cyg & 37 & 73 & 69 & 46 & 37 & 60 & 64 & 41 \\
\hline KY Cyg & 40 & 65 & 61 & 48 & 36 & 56 & 60 & 42 \\
\hline NML Cyg & 66 & 79 & 73 & 73 & 60 & 80 & 79 & 70 \\
\hline$\mu$ Сep & 44 & 76 & 76 & 54 & 42 & 62 & 69 & 47 \\
\hline PZ Cas & 41 & 66 & 61 & 49 & 37 & 56 & 56 & 40 \\
\hline TZ Cas & 38 & 70 & 68 & 41 & 32 & 56 & 60 & 33 \\
\hline \multicolumn{9}{|l|}{ (b) $M$ giants } \\
\hline W And & 30 & 77 & 64 & 38 & 33 & 58 & 61 & 35 \\
\hline KU And & 65 & 81 & 81 & 60 & 62 & 72 & 76 & 77 \\
\hline T Cas & 45 & 81 & 74 & 49 & 40 & 66 & 71 & 43 \\
\hline IK Tau & 68 & 91 & 80 & 65 & 64 & 85 & 89 & 64 \\
\hline UX Cyg & 64 & 85 & 78 & 62 & 56 & 75 & 80 & 59 \\
\hline \multicolumn{9}{|l|}{ (c) $S$ stars } \\
\hline $\mathrm{R}$ And & 31 & 72 & 69 & 34 & 31 & 53 & 59 & 36 \\
\hline W Aql & 35 & 68 & 66 & 39 & 35 & 52 & 53 & 38 \\
\hline AD Cyg & 27 & 68 & 66 & 40 & 22 & 57 & 56 & 27 \\
\hline \multicolumn{9}{|c|}{ (d) Carbon stars } \\
\hline HV Cas & 79 & 84 & 84 & 86 & 80 & 89 & 86 & 82 \\
\hline V466 Per & 50 & 70 & 74 & 56 & 48 & 65 & 63 & 51 \\
\hline TT Tau & 34 & 65 & 67 & 46 & 35 & 61 & 63 & 41 \\
\hline V Cyg & 70 & 87 & 84 & 83 & 74 & 90 & 89 & 79 \\
\hline V460 Cyg & 39 & 70 & 73 & 48 & 38 & 59 & 59 & 39 \\
\hline RV Cyg & 52 & 73 & 73 & 61 & 50 & 70 & 66 & 57 \\
\hline \multicolumn{9}{|c|}{ (e) $R V$ Tauri variables } \\
\hline RV Tau & 80 & 89 & 92 & 97 & 80 & 99 & 100 & 86 \\
\hline AC Her & 93 & 96 & 95 & 103 & 93 & 102 & 100 & 95 \\
\hline
\end{tabular}

${ }^{a}$ Blended with $v=2-0 R 71 ;{ }^{b}$ blended with $v=3-1 R 17$ and ${ }^{13} \mathrm{CO} v=2-0 B H$

${ }^{c}$ blended with $v=2-0 R 13 ;{ }^{d}$ blended with $v=3-1 R 12$

$K$-[12] color (Figs. 30-33) is in fact a symptom of the large variation in atmospheric dynamical structure with time. This connection reinforces the case for the CO bands as diagnostics for hydrodynamic models.

\subsection{Spectral variability}

There is previous evidence for variability in $\mathrm{CO}$ and other molecular bands in the near IR spectra of cool giants which, it has been thought, were modulated with the phase of the light cycle, though the connection with phase is more an assumption than a demonstrated correlation. Frogel (1971) conducted an extensive study of 18 Mira variables, mostly of late-M spectral type. His data typically extend over one period of stellar variation. Changes in $\mathrm{CO}$ absorption strength were detected for most of the stars. The bands were systematically weaker at stellar temperature minima than at maxima, but there was a wide range of behavior relative to precise phase of the light cycle. Time variations in the CO 2-0 bandhead of the S star $\chi$ Cyg were reported by Wallace \& Hinkle (1997), who found that the absorption depth varied over the light cycle from 0.49 to 0.66 of the continuum when observed at $R=2700$. Aringer et al. (1999) found that for a sample of 8 Miras, the equivalent width of the $\mathrm{SiO}$ first overtone bandheads varied systematically with phase.

We plotted the $\mathrm{CO} 2-0$ bandhead intensity versus phase for the 9 Miras in our sample with allegedly accurate periods, where the phase was determined from periods and reference epochs in the GCVS. There is no evidence of a correlation in our data. Three factors could mask any modulation of the $\mathrm{CO}$ features with the light cycle however. First, there may be a large intrinsic spread in the CO 2-0 bandhead depth from star to star, both in the mean and the extreme values over a cycle. Second, the phases we derived (see Table 1) may have some accumulated error over the time interval from the reference epoch, or the period may have changed or be modulated by a 


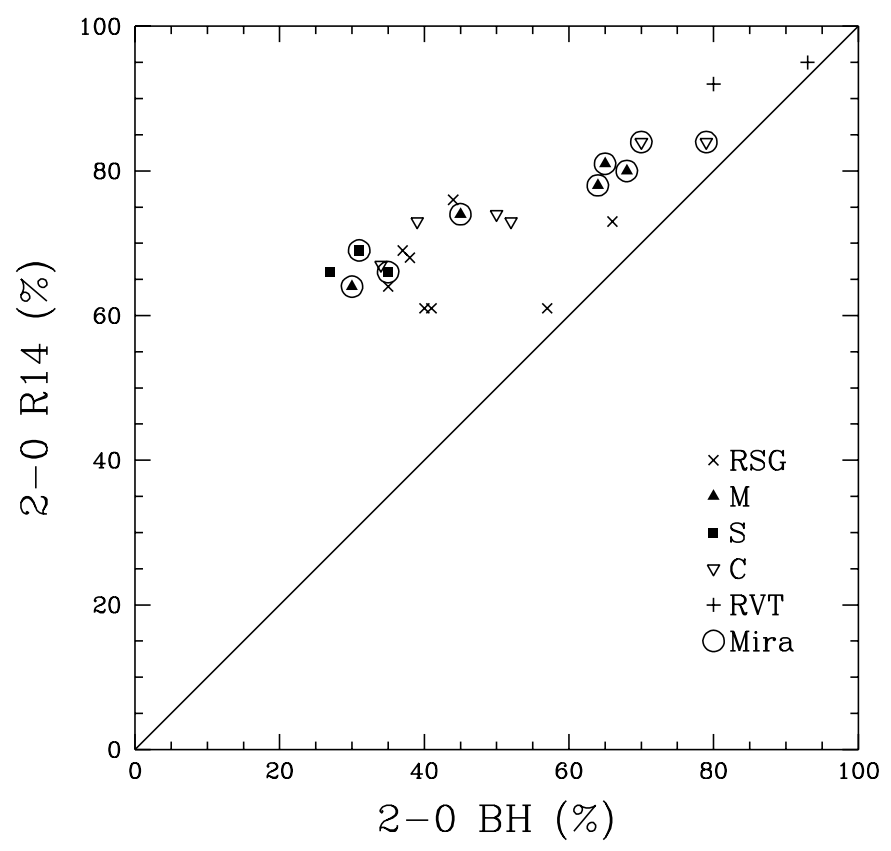

Fig. 27. $v=2-0$ R14 intensity vs. $2-0$ bandhead intensity. Symbols denote red supergiants, $\mathrm{M}$ giants, $\mathrm{S}$ stars, carbon stars, and RV Tauri variables as indicated. Circles indicate Mira variables.

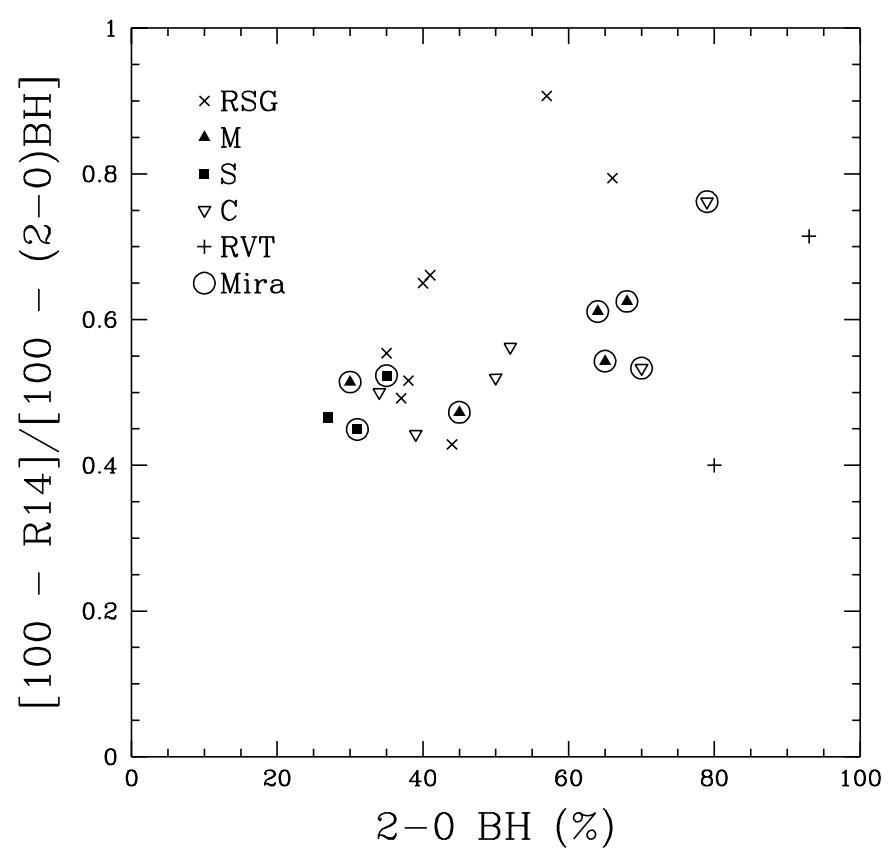

Fig. 28. Ratio of R14 absorption depth to (2-0) bandhead absorption depth, vs. (2-0) bandhead intensity. Symbols as in Fig. 27.

second period. Third, long-term cycle-to-cycle variations in the atmospheric structure may also modulate the $\mathrm{CO}$ absorption lines. Recent theoretical calculations find that the envelope structure for some models may change over a timescale longer than the stellar pulsation. Hofmann et al. (1998) see such cycle-to-cycle behavior in their pulsational models for M-type Miras. Winters et al. (2000) find that hydrodynamic models for the carbon star IRC+10216

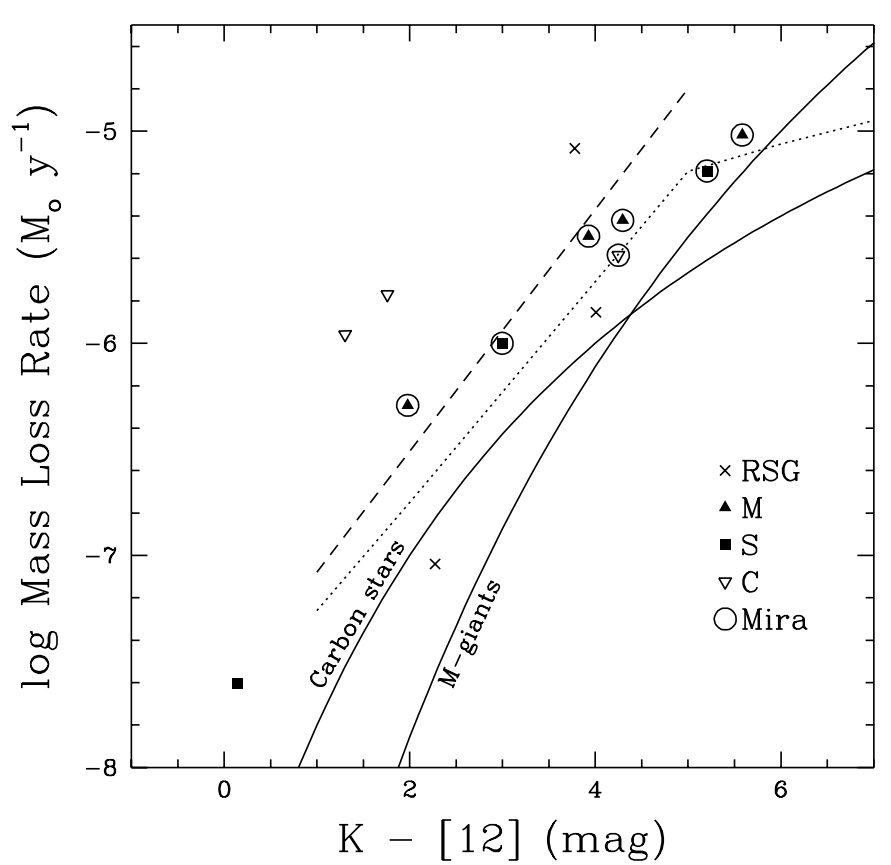

Fig. 29. Gas mass loss rate from $\mathrm{CO}$ mm-wavelength emission lines (see Table 1), vs. K-[12] color. Symbols as in Fig. 27. Dotted line shows mean relation found by Whitelock et al. (1994) for Miras in South Galactic Cap. Dashed line is for red supergiants assuming a gas-to-dust ratio of 200 and the $\dot{M}_{\text {dust }}$ vs. $K$-[12] relation from Josselin et al. (2000). Labelled curves show relations derived by LeBertre \& Winters (1998) for carbon-rich and oxygen-rich (M-type) Miras.

show individual CO lines varying in shape and intensity over several light cycles, as a consequence of episodes of dust shell formation and ejection. These models suggest that there may be no simple correlation in spectral variations with the light cycle, at least for the Miras. Rather, longer term variations are to be expected, as the observations of IRC +10216 presented by Winters et al. seem to indicate.

Comparison of the spectra of the 4 supergiants in our sample which were also observed by Wallace \& Hinkle (1997) shows no evidence for variability. The $2-0$ and $3-$ 1 bandheads are resolved in both data sets, and in all 4 stars, the depth and width of the bandheads are the same within the noise for each pair of spectra. The individual $R$-branch lines differ only to the degree expected due to the different reolutions of the two sets of spectra. The Wallace \& Hinkle (1997) spectra were obtained in 1981 June ( $\mu$ Cep and SU Per) and 1984 April (KY Cyg and PZ Cas), so on a timescale of more than a decade, we see no evidence for spectral variability in the CO bands for these red supergiants.

The two RV Tauri variables, which are believed to be in a post-AGB evolutionary stage, are more interesting candidates for spectral variability. Oudmaijer et al. (1995) note that 3 of 5 known post-AGB stars with $2.3 \mu \mathrm{m}$ CO in emission show spectral variability. One of these is AC Her (= HD 170756). Oudmaijer et al. argue that the CO overtone bands are in emission at the optical ( $V$-band) 


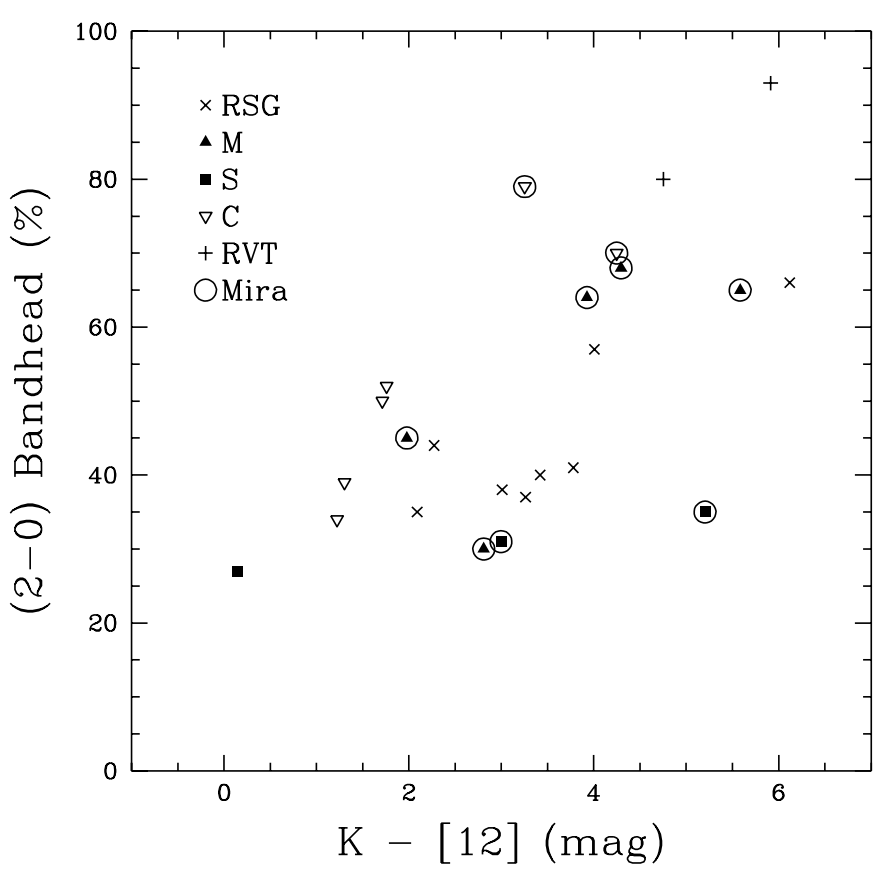

Fig. 30. (2-0) bandhead intensity vs. $K$-[12] color. Symbols as in Fig. 27.

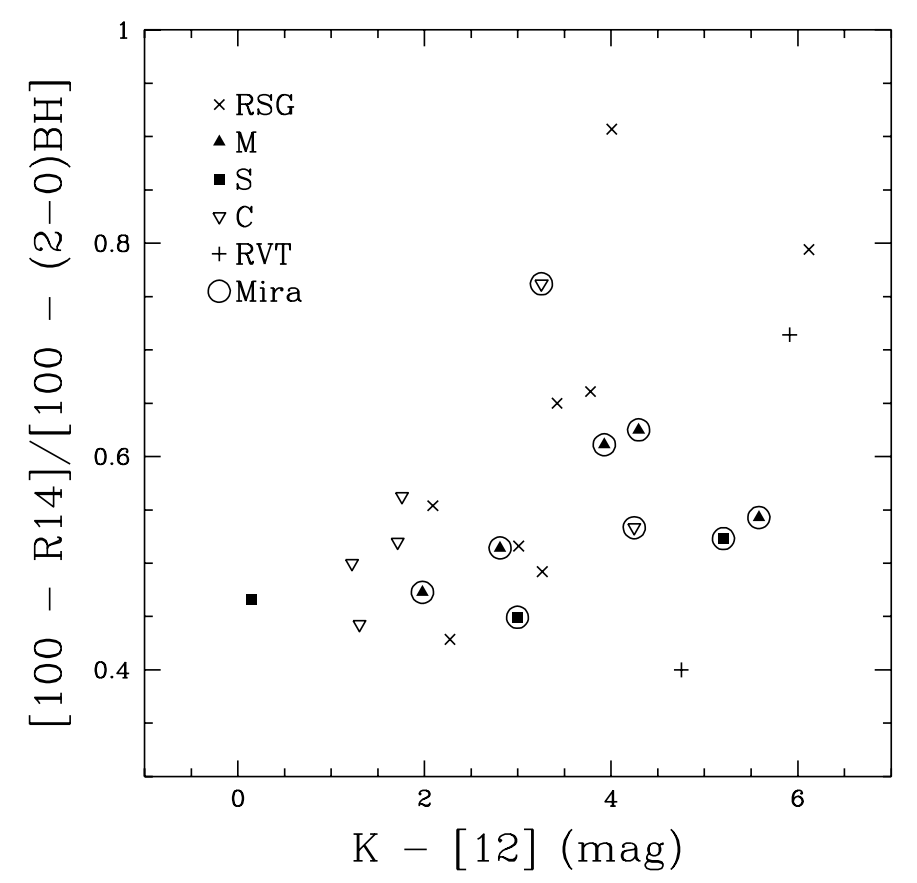

Fig. 31. Ratio of $R 14$ absorption depth to (2-0) bandhead absorption depth), vs. K-[12] color. Symbols as in Fig. 27.

minimum, and go into absorption during the decline after secondary maximum. Their data clearly show dramatic changes in the $\mathrm{CO}$ first overtone spectra, but the sampling of the light cycle is too sparse and irregular to draw firm conclusions. In our spectrum of AC Her (Fig. 25), which was taken at about phase 0.3 near or just after the secondary minimum in the light cycle, the CO 2-1 bandhead depth is about $7 \%$ of the continuum (i.e., $I(2-0 B H)$ $=0.93)$. This value is only half the absorption depth

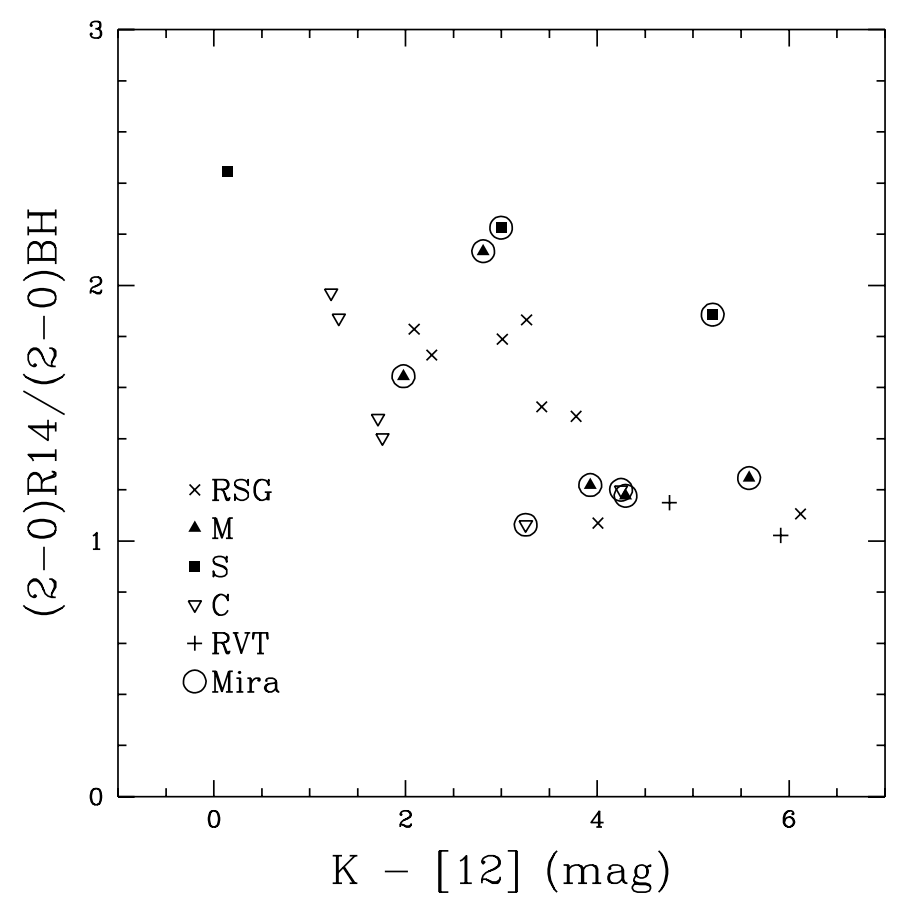

Fig. 32. Ratio of $R 14 /(2-0)$ bandhead intensities vs. $K$-[12] color. Symbols as in Fig. 27.

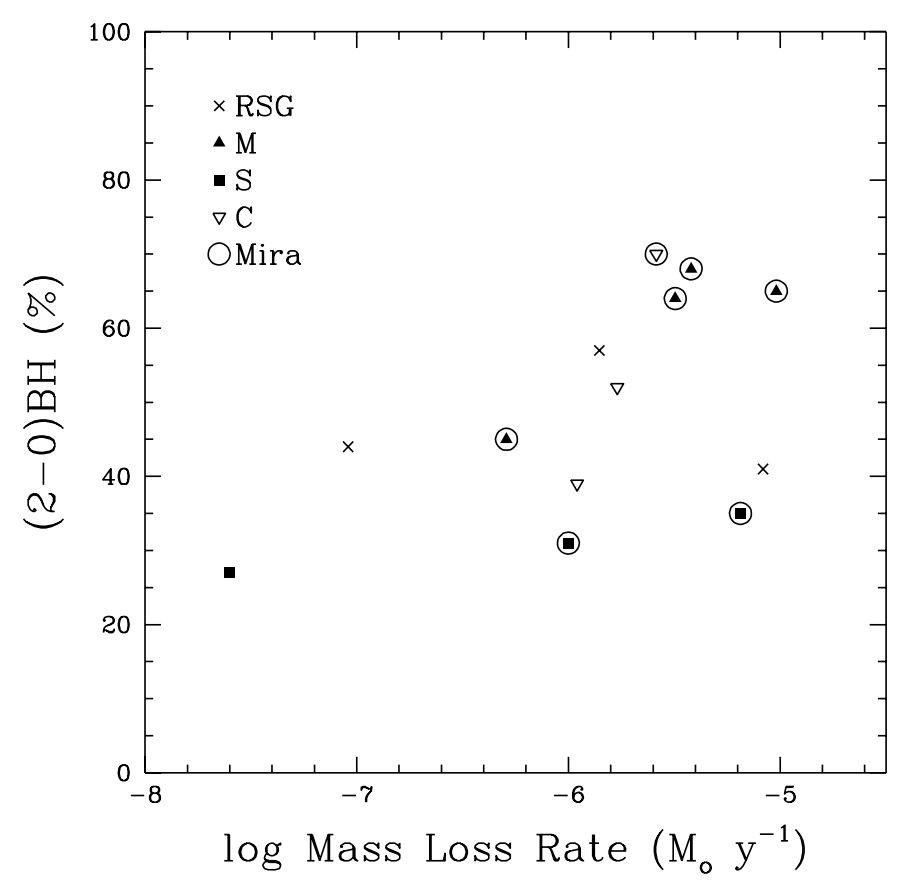

Fig. 33. (2-0) bandhead intensity vs. gas mass loss rate. Symbols as in Fig. 27.

measured by Oudmaijer et al. (1995) for a spectrum taken at phase 0.90 . Figure 25 also shows an indication of weak emission in the vicinity of the $2-0 R 0$ and $P 1$ lines, at a few per cent of the continuum, but the bandheads are definitely in absorption. Our spectrum evidently shows the CO bands in a state intermediate between the deep CO absorption and emission reported by Oudmaijer et al. They argued that the variability was a result of episodic mass ejection during the pulsational cycle, with emission 
occurring just after ejection of gas while it is still close to the star in a dense warm state. Post-AGB mass loss is probably closely related to the formation and shaping of planetary nebulae (e.g., Kwok 1993), but the physical mechanisms are poorly understood. Well-sampled monitoring of the $2.3 \mu \mathrm{m}$ CO bands over the light cycle of AC Her and related stars could be a very useful diagnostic for the mass loss. The relatively short periods $\left(\sim 70^{\mathrm{d}}\right)$ of these stars would help make such a monitoring program practical.

\subsection{CO isotopomers, atomic lines, and other molecular lines}

The $2-0$ bandhead of ${ }^{13} \mathrm{CO}$ at $2.3448 \mu \mathrm{m}$ is a relatively prominent feature in most of the spectra in Figs. 2-25. It is important to note, however, that at a resolution of $R=3500$ the bandhead is affected by other lines close in wavelength. The 2-0 $R 0$ and $3-1 R 17$ lines of the main $\mathrm{CO}$ isotopomer, which are almost coincident, lie within one resolution element of the ${ }^{13} \mathrm{CO} 2-0$ bandhead. The individual $R$-branch lines of the main isotopomer are typically quite strong, and these two clearly contribute to the absorption at the wavelength of the ${ }^{13} \mathrm{CO}$ bandhead. Interpreting the ${ }^{13} \mathrm{CO} 2-0$ bandhead strength in terms of a ${ }^{12} \mathrm{CO} /{ }^{13} \mathrm{CO}$ abundance ratio must include a calculation of the main isotopomer $R$-branch lines near the bandhead.

In this connection, there is also a very close coincidence between the ${ }^{13} \mathrm{CO} 2-0$ bandhead and a line of Ti I at $2.34479 \mu \mathrm{m}$. This line is not separated from the ${ }^{13} \mathrm{CO}$ bandhead even at very high resolution in the spectrum of Arcturus by Hinkle et al. (1995). Another Ti I line at $2.2970 \mu \mathrm{m}$ is prominent on the side of the CO $2-0$ bandhead in most of our spectra, typically absorbing $\sim 5 \%$ of the continuum at $R=3500$. The strength of this line suggests that the $\mathrm{Ti}$ I line coincident with the ${ }^{13} \mathrm{CO} 2-$ 0 bandhead also contributes significantly to the observed absorption feature. As with the $R$-branch lines discussed above, the effect of Ti I absorption must be included in comparing the ${ }^{13} \mathrm{CO} 2-0$ bandhead with ${ }^{12} \mathrm{CO}$.

The spectra of our sample of carbon stars (Figs. 1822) show remarkable variations in the depth and shapes of the CO $2-0$ bandheads and main $R$-branch lines (compare for example HV Cas - Fig. 18 - with V460 Cyg Fig. 22). The main features in all cases do appear to be the $\mathrm{CO}$ overtone band lines, but it is clear that the bands are modulated by other molecules with features in the observed range of wavelengths. It is well known that carbon stars have a rich organic chemistry in their atmospheres and circumstellar envelopes. A recent study of ISO-SWS spectra by Jørgensen et al. (2000) identified $\mathrm{C}_{2}, \mathrm{CN}, \mathrm{CH}$, $\mathrm{CS}, \mathrm{HCN}, \mathrm{C}_{3}$, and $\mathrm{C}_{2} \mathrm{H}_{2}$ in the spectra of V460 Cyg over the 2.4 to $45 \mu \mathrm{m}$ range. The theoretical models of Helling et al. (2000) for carbon-rich AGB stars indicate that the main contributors to molecular opacity in the $2.3 \mu \mathrm{m}$ region, besides $\mathrm{CO}$, include $\mathrm{C}_{2}, \mathrm{CN}, \mathrm{C}_{3}$, and $\mathrm{HCN}$. The contribution of each to the absorption lines in our observed wavelength band depends on the temperature and density structure, and on the $\mathrm{C} / \mathrm{O}$ ratio. Differences in these properties among our sample of carbon stars could easily explain the variations in the observed spectra. We have not attempted to identify other molecular lines in these spectra, and in fact most of the individual absorption features can be identified with $\mathrm{CO}$ lines. The differences in the detailed shapes of the $R$-branch lines in the $2-0$ band as compared with the very regular structure in the $M-$ giants or supergiants, however, indicates the presence of lines from other carbon-bearing molecules. Accurate models for the carbon star spectra in this wavelength region must include these species in addition to CO.

\section{Conclusions}

We have presented spectra covering the wavelength range 2.28 to $2.36 \mu \mathrm{m}$ for a sample of $24 \mathrm{cool}$ evolved stars. The sample comprises $8 \mathrm{M}$ supergiants, $5 \mathrm{M}$ giants, $3 \mathrm{~S}$ stars, 6 carbon stars, and 2 RV Tauri variables. The wavelengths covered include the main parts of the ${ }^{12} \mathrm{C}^{16} \mathrm{O} v=2-0$ and $3-1$ overtone bands, as well as the $v=4-2$ and ${ }^{13} \mathrm{CO} v=$ $2-0$ bandhead regions. The observed transitions arise from a wide range of energy levels extending from the ground state to $E / k>20000 \mathrm{~K}$. The spectra have a resolution of $\Delta \lambda=0.0007 \mu \mathrm{m}$, or $R=3500$. CO lines dominate the spectrum for all the stars observed, and at this resolution most of the observed features can be identified with individual CO $R$ - or $P$-branch lines or blends. We looked for correlations between the intensities of various $\mathrm{CO}$ absorption line features and with other stellar properties, including IR colors and mass loss rates. Two of the most useful CO line features appear to be the $2-0 R 14$ line which is well-resolved from any other $\mathrm{CO}$ or atomic features; and the CO 2-0 bandhead, which, as the shortest wavelength of the first overtone bandheads, is not blended with any other CO lines nor any known atomic lines. The most significant conclusions are:

(1) The 2-0 R14 intensity shows a non-linear correlation with the $2-0$ bandhead intensity, with an apparent leveling off in $R 14$ for the deepest 2-0 bandheads (see Fig. 27).

(2) The ratio of the $R 14$ line depth (below the continuum) to the $2-0$ bandhead depth converges to a value of $\sim 0.5$ for the deepest $2-0$ bandheads $(I(2-0 B H)<40 \%)$. Stars with shallower $2-0$ bandheads $(I(2-0 B H)>50 \%)$ show a large scatter in the ratio of line depths (Fig. 28).

(3) The published gas mass loss rates for both the AGB stars and the red supergiants in our sample correlate with the $K$-[12] color, consistent with other studies. The intensity of the 2-0 bandhead shows a trend with $K$-[12] color such that the reddest stars $(K-[12]>3 \mathrm{mag})$ exhibit a wide range in $2-0$ bandhead depth, while the least reddened have the deepest $2-0$ bandheads, with a small range of variation from star to star.

(4) Similarly, the reddest stars $(K-[12]>3 \mathrm{mag})$ exhibit a large range in the ratio of the depth of the $2-0 R 14$ line to the 2-0 bandhead depth. Stars with less reddening 
$(K-[12]<3 \mathrm{mag})$ show about one-third the range in this ratio compared to the redder sample. If we interpret the $K$-[12] color in terms of a mass loss rate, the data imply that stars with $\dot{M}_{\text {gas }}<5 \times 10^{-7} M_{\odot} \mathrm{y}^{-1}$ exhibit a much narrower range in the relative strengths of $\mathrm{CO} 2-0$ band features than stars with higher mass loss rates.

(5) The wide range in CO feature intensities and intensity ratios for the most reddened stars suggests that dust continuum is not filling in the absorption, at least not in every case, but this effect needs careful modelling in any synthetic spectrum calculation.

(6) There is no evidence for a correlation of the CO band strengths with phase of the light cycle for the Miras, but intrinsic differences from star to star, or cycle-to-cycle variations, could mask any real effect. Our spectrum of AC Her, a post-AGB star, differs significantly from comparable spectra taken at other epochs. Well-sampled monitoring of selected stars is needed to determine the extent of spectral variability.

(7) Although the ${ }^{12} \mathrm{C}^{16} \mathrm{O}$ overtone bands dominate the observed spectra, we note that other atomic and molecular species are present and need to be considered. First, the ${ }^{13} \mathrm{C}^{16} \mathrm{O} 2-0$ bandhead is closely blended with an atomic line of TiI, and with the $3-1 R 17$ and $2-0 R 0$ lines of ${ }^{12} \mathrm{C}^{16} \mathrm{O}$. The observed ${ }^{13} \mathrm{C}^{16} \mathrm{O} 2-0$ bandhead depth will be affected by these features. Second, the carbon star spectra show significant modulation by other molecular lines in this wavelength region. The likely contributors include $\mathrm{C}_{2}$, $\mathrm{CN}, \mathrm{CH}, \mathrm{CS}, \mathrm{HCN}, \mathrm{C}_{3}$, and $\mathrm{C}_{2} \mathrm{H}_{2}$. We have not attempted to identify features of these molecules, and in fact almost all of the discrete features we observe in the carbon star spectra can be identified with the CO overtone bands. Precise modelling of this spectral region for carbon stars, however, will require that these other molecular species be included.

The range in spectral properties that we observe for this sample of cool giant and supergiant stars implies that there are significant differences in atmospheric structure. Spectra of the CO molecule should be good diagnostics for the structure of the extended atmospheres of these pulsating, mass-losing stars. Such data may be used to test hydrodynamic models which are being developed with more realistic treatment of stellar pulsations, shocks, dust formation, and molecular chemistry.

Acknowledgements. JHB thanks C. Engelbracht for invaluable help with data processing; and acknowledges support from the U.S. National Science Foundation through grants AST9618523 and AST-9987408. We also thank the referee, Dr. M. Scholz, and Dr. J. M. Winters for helpful comments. This research has made use of the SIMBAD database, operated at CDS, Strasbourg, France.

\section{References}

Aringer, B., Höfner, S., Wiedemann, G., et al. 1999, A\&A, 342, 799
Beichman, C. A., et al. 1985, IRAS Explanatory Supplement, NASA RP-1190

Bessell, M. S., Scholz, M., \& Wood, P. R. 1996, A\&A, 307, 481

Bieging, J. H., \& Latter, W. B. 1994, ApJ, 422, 765

Duari, D., Cherchneff, I., \& Willacy, K. 1999, A\&A, 341, L47

Farrenq, R., Guelachvili, G., Sauval, A. J., Grevesse, N., \& Farmer, C. B. 1991, J. Mol. Spec., 149, 375

Feast, M. W., \& Whitelock, P. A. 2000, MNRAS, 317, 460

Fleischer, A. J., Gauger, A., \& Sedlmayr, E. 1992, A\&A, 266, 321

Frogel, J. A. 1971, Ph.D. Dissertation, California Institute of Technology (Ann Arbor: University Microfilms)

Gezari, D. Y., Pitts, P. S., \& Schmitz, M. 1999, Catalog of Infrared Observations, Edition 5 (NASA Goddard Space Flight Center, available at http://adc.gsfc.nasa.gov/adc-cgi/cat.pl?/catalogs/2/2225/)

Helling, Ch., Winters, J. M., \& Sedlmayr, E. 2000, A\&A, 358, 651

Hinkle, K. H., Hall, D. N. B., \& Ridgway, S. T. 1982, ApJ, 252, 697

Hinkle, K. H., Scharlach, W. W. G., \& Hall, D. N. B. 1984, ApJS, 56, R1

Hinkle, K., Wallace, L., \& Livingston, W. 1995, Infrared Atlas of the Arcturus Spectrum, 0.9-5.3 microns (Provo: Astron. Soc. of the Pacific)

Höfner, S. 1999, A\&A, 346, L9

Höfner, S., Jørgensen, U. G., Loidl, R., \& Aringer, B. 1998, A\&A, 340, 497

Hofmann, K.-H., Scholz, M., \& Wood, P. R. 1998, A\&A, 339, 846

Hron, J., Loidl, R., Höfner, S., et al. 1998, A\&A, 335, L69

Jørgensen, U. G., Hron, J., \& Loidl, R. 2000, A\&A, 356, 253

Josselin, E., Blommaert, J., Groenewegen, M., Omont, A., \& Li, F. 2000, A\&A, 357, 225

Kholopov, P. N. 1985, General Catalogue of Variable Stars (Moscow: Nauka Publ. House) (GCVS)

Kleinmann, S. G., \& Hall, D. N. B. 1986, ApJS, 62, 501

Kwok, S. 1993, ARA\&A, 31, 63

Le Bertre, T. 1997, A\&A, 324, 1059

Le Bertre, T., \& Winters, J. M. 1998, A\&A, 334, 173

Loidl, R., Höfner, S., Jørgensen, U. G., \& Aringer, B. 1999, A\&A, 342, 531

Loup, C., Forveille, T., Omont, A., \& Paul, J. F. 1993, A\&AS, 99, 291

Neri, R., Kahane, C., Lucas, R., Bujarrabal, V., \& Loup, C. 1998, A\&AS, 130, 1

Neugebauer, G., \& Leighton, R. B. 1969, Two Micron Sky Survey (NASA SP-3047)

Oudmaijer, R. D., Waters, L., van der Veen, W., \& Geballe, T. 1995, A\&A, 299, 69

Scholz, M., \& Wood, P. R. 2000, A\&A, 362, 1065

Wallace, L., \& Hinkle, K. 1997, ApJS, 111, 445

Whitelock, P., Menzies, J., Feast, M., et al. 1994, MNRAS, 267,711

Willacy, K., \& Cherchneff, I. 1998, A\&A, 330, 676

Williams, D. M., Thompson, C. L., Rieke, G. H., \& Montgomery, E. F. 1993, Proc. SPIE, 1946, 482

Winters, J. M., Fleischer, A. J., Le Bertre, T., \& Sedlmayr, E. 1997, A\&A, 326, 305

Winters, J. M., Keady, J. J., Gauger, A., \& Sada, P. V. 2000, A\&A, 359, 651 